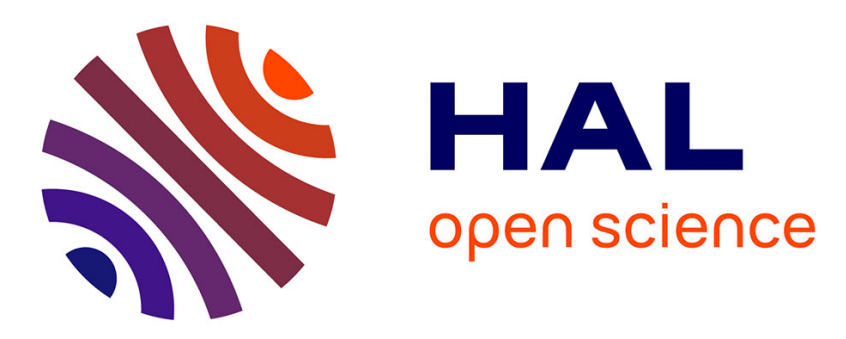

\title{
Automatic Color Detection-Based Method Applied to Sentinel-1 SAR Images for Snow Avalanche Debris Monitoring
}

Anna Karas, Fatima Karbou, Sophie Giffard-Roisin, Philippe Durand, Nicolas Eckert

\section{To cite this version:}

Anna Karas, Fatima Karbou, Sophie Giffard-Roisin, Philippe Durand, Nicolas Eckert. Automatic Color Detection-Based Method Applied to Sentinel-1 SAR Images for Snow Avalanche Debris Monitoring. IEEE Transactions on Geoscience and Remote Sensing, In press, 60, pp.5219117. 10.1109/TGRS.2021.3131853 . hal-03467334

\section{HAL Id: hal-03467334 https://hal.science/hal-03467334}

Submitted on 6 Dec 2021

HAL is a multi-disciplinary open access archive for the deposit and dissemination of scientific research documents, whether they are published or not. The documents may come from teaching and research institutions in France or abroad, or from public or private research centers.
L'archive ouverte pluridisciplinaire $\mathbf{H A L}$, est destinée au dépôt et à la diffusion de documents scientifiques de niveau recherche, publiés ou non, émanant des établissements d'enseignement et de recherche français ou étrangers, des laboratoires publics ou privés. 


\title{
Automatic Color Detection-Based Method Applied to Sentinel-1 SAR Images for Snow Avalanche Debris Monitoring
}

\author{
Anna Karas, Fatima Karbou, Sophie Giffard-Roisin, Philippe Durand, Nicolas Eckert
}

\begin{abstract}
In this study, we develop a novel method to automatically detect areas of snow avalanche debris using a color space segmentation technique applied to SAR image time series through January 2018 in the Swiss Alps. Debris avalanche zones are detected assuming that these areas are characterised by a significant and localised increase in SAR signal relative to the surrounding environment. We undertake a sensitivity study by calculating debris products by varying the $D$-M reference images (a stable reference image taken several weeks before the event). We examine the results according to the direction of the orbit, the characteristics of the terrain (slope, altitude, orientation) and also by evaluating the relevance of the detection with the help of an independent SPOT database ([1]) including 18,737 avalanche events. Small avalanches are not detected by SAR images and depending on the orientation of the terrain some avalanches are not detected by either the ascending or the descending orbit. The detection results vary with the reference image; best detection results are obtained with some selected individual dates with almost $70 \%$ of verified avalanche events using the ascending orbit.
\end{abstract}

Index Terms-Automatic detection, Color space, Random Forest, SAR, Sentinel-1, Segmentation, Snow Avalanche

\section{INTRODUCTION}

$\mathbf{S}$ NOW avalanches represent a serious hazard in the mountains, with direct consequences on human lives and considerable damage to the dwellings and infrastructure of the communities concerned. Large-scale and regular avalanche monitoring is essential for the needs of snowpack stability evaluation and avalanche forecasting. Field monitoring of snow avalanches is generally fairly sparse and limited in time and space. This is particularly true for areas that are difficult to access. It should also be noted that periods of high danger level of avalanches are often associated with very bad weather conditions, which further limits field observations.

By contrast, with satellite based remote sensing observations, it is becoming increasingly conceivable to carry out reliable and regular monitoring of areas affected by snow avalanche debris ([2]).

Optical images from both aircraft and satellites have been used to map avalanche events with high and very high spatial

A. Karas, F. Karbou are with Univ. Grenoble Alpes, Université de Toulouse, Météo-France, CNRS, CNRM, Centre d'Études de la Neige, Grenoble, France (Corresponding author: anna.karas@meteo.fr).

S. Giffard-Roisin is with ISTerre; Univ. Grenoble Alpes, Univ. Savoie Mont Blanc, CNRS, IRD, IFSTTAR Grenoble, France

$\mathrm{Ph}$. Durand is with CNES, Toulouse, France.

N. Eckert is with UR ETNA, INRAE, Université Grenoble Alpes, France resolution, but these images require generally cloud-free conditions and can be used over some specific target territories ([3], [4], [5], among others). [5] presented an automatic method of texture classification on very high resolution optical imagery. A lot of studies are based on object-based image analysis. [3] used object-based classification and combined directional, textural and spectral information. [4] generated avalanche maps with an object-oriented image interpretation approach (segmentation and classification) within very high resolution panchromatic optical imagery. [6] developed an automatic object-based approach to detect avalanches deposits on near-infrared images. More recently, [1] performed a systematic avalanche mapping on one SPOT6 image over the entire Swiss Alps. 18,737 avalanche events where mapped during the extreme avalanche period of January 2018. The description of the generation of this SPOT6 database is presented by [7]. [8] confirmed the high potential of SPOT6-7 images in mapping avalanche outlines by using ground truth validation points. They studied two avalanche activity periods in 2018 and 2019, in a $180 \mathrm{~km}^{2}$ area around Davos, and found: a Probability of Detection of $74 \%$ (POD, events correctly detected by a human on the satellite data) and a Positive Predictive Value (percentage of identified events by manual detection that were an avalanche event) of $88 \%$.

While the use of optical data is limited by cloud cover, which should be as low as possible, usable data from radar satellites are independent of illumination and weather conditions ([9], [10]). Moreover, optical remote sensing imagery can be expensive and only available on request for specific areas, as is the case of optical SPOT6-7 ([8]). Sentinel-2 is free of charge, but [8] found it not suitable for avalanche mapping. The use of Synthetic Aperture Radar (SAR) is increasingly used in avalanche detections to obtain a continuity of observations. Sentinel-1 is publicly available while some satellite data cannot provide systematic coverage but only upon request for scientists such as TerraSAR-X. The first SAR avalanche detections were undertaken by [10] using ERS-1 ans ERS-2 C-band SAR data. A change detection method was used to identify high backscatter coefficient in multi-temporal SAR images. The main idea behind the detection of debris by SAR imagery is that snow avalanche debris exhibit a higher localised radar backscatter compared to the surrounding snow due to increased surface roughness and increased snow depth at the avalanche site ([11], [9]). [10] used three images for the RGB composite image, the multi-temporal combination of SAR images: one image taken one month before the event (in 
the $\mathrm{R}$ channel), during the avalanche activity period (in the $\mathrm{G}$ channel) and one without snow (end of September, in the B channel). Thus, the local backscatter increase can be seen in green in a RGB composite image.

First studies on SAR avalanche detection often considered one radar image acquired after the event or two images, one before and one taken after the event as a difference image ([12], [9], [13], among others). We can also note that most of the studies, that used change detection methods applied to a series of SAR images, masked complex areas for the detection (layover, forest, etc.) ([14], [15], [16]). With single backscatter images, the detection was based on enhancement methods to improve the backscatter contrast between avalanche deposits and the surrounding snow ([12], [17], [9]). By using two SAR images, the detection method was based on a backscatter change between the reference image (taken few days before the event) and the activity image (taken just after the event) ([13], [9], [16]). Different SAR satellites were used. [13] showed that TerraSAR-X could be a reliable tool to manually map avalanches. [17] demonstrated that manual avalanche detection can be undertaken using Radarsat 2 measurements. [2] later showed successful results using manual avalanche detection on Sentinel-1 images. More recently, [16] compared field recordings with manual detections and obtained a Probability of Detection of $77.3 \%$ using Sentinel-1 SAR imagery.

However, manual mapping is time-consuming which supports the development of automatic detection methods. [18] tested an automatic detection method by combining a change detection approach with a K-means classification method. They have shown satisfactory detection rates when the method was applied under typical operational monitoring circumstances. This method was also used by [15], based on a backscatter change within a 6-days period on Sentinel-1 images. [16] developed a near-real time automatic avalanche monitoring system that was first applied pre-operationally on three regions in Norway by the Norwegian Avalanche Warning service, also using Sentinel-1 SAR imagery. From winter 2020-2021, it is daily used in all Norwegian forecasting regions ([19]). Thus, forecasters can produce feedback on avalanche risk. [16] compared this automatic method to field observations and manual detection: the POD were $57 \%$ and $79 \%$, respectively.

[20] compared avalanche mapping with optical and SAR images: radar Sentinel-1 with TerraSAR-X and optical SPOT67 images. The comparison of detection methods was also made: single image avalanche detection, two-image composite avalanche detection, multi-orbital composite image (one RGB composite image made of a combination of 12 images taken before the avalanche activity period and 12 images taken after the events) and automatic avalanche detection. [20] found that change detection is more sensitive than single avalanche detection. The change detection with TerraSAR-X images detected more avalanche events than with the Sentinel1 (small avalanches were missed due to the lower resolution of Sentinel-1 compared to TerraSAR-X). [8] used similar pre-processing steps of [20] and compared the reliability of avalanche detections of: SPOT-6, Sentinel-1 and optical Sentinel-2 images. [20] and [8] studies found that Sentinel-
1 is effective in mapping avalanches (significant spatial and temporal coverage) despite the fact that these observations do not help to detect avalanches of small and medium sizes. [8] obtained a POD of 0.27 using Sentinel-1 manual mapping. The authors found a lower POD than for the SPOT mapping because of the majority of medium and large size avalanches.

To minimise the effects of geometric distortions, [21] suggests merging the ascending and descending orbits with the local resolution weighting (LRW). This is based on applying a weighted average according to incidence angles to merge acquisitions from different orbits. In the study of [20], the authors showed that the use of LRW improves avalanche visibility by weighting the slopes opposite the radar more strongly. [8] used similar SAR processing steps and used pre and post avalanche event LRW image differences to map areas associated with an increase in the SAR backscatter signal. [22] proved that a segmentation with a fixed threshold was more accurate on LRW images $(F 1=0.75)$ than on unweighted average orbits $(\mathrm{F} 1=0.68)$ and on single orbit images $(\mathrm{F} 1=$ $0.5)$.

The change detection method using three different SAR images was recently used to manually detect avalanche debris in the French Alps. In the study of [23], time series of Sentinel1 images were studied. [23] investigated the possibility to synthesise and visualise the detected avalanche activity at a regional scale (e.g. $500 \mathrm{~km}^{2}$ ) by aggregating the detected avalanche activity according to some terrain characteristics (elevation, slope, orientation).

Recent studies also evaluated the potential of machine learning approaches such as Random Forests and Convolutional Neural Networks using Sentinel-1 imagery. In the work of [24], [25] and [26], a binary classification of avalanches was realised. [25] and [27] were mostly based on manual interpretation, whereas in [26], an independent avalanche inventory was used. This last study explored the question of the potential of deep learning methods which could help improve the detection of avalanche debris. More recently, [22] trained and tested a deep neural U-Net using manual mapped avalanches outlines and obtained a high detection score $(\mathrm{F} 1=$ 0.81). Random Forest is a machine learning approach and is very effective in classification ([28]). The results of [29] are promising to automatically detect and map snow avalanches. [30] used Random Forest to classify and predict avalanche activity in the French Alps by taking into account snow and meteorological conditions. Random Forest was already used in various and multiple applications and is especially well suited for limited data size : it does not overfit, it is fast and also robust with respect to noise ([28]).

Although the potential of Sentinel-1 SAR data is promising for snow avalanche mapping, their exploitation still raises significant scientific challenges. Meteorological conditions can impact the snow pack (wind, change in temperature, precipitation, etc.) and the heterogeneity of mountainous terrain makes it all the more difficult to study the SAR signal and its interaction with the snow cover. Moreover, the side-looking radar and the complex mountainous terrain cause geometric distortions in the radar image like foreshortening, layover and shadows. In-situ observation datasets are limited by the terrain 
accessibility and are realised punctually for specific avalanche paths.

In this work, a new automatic debris detection method is evaluated using part of the [1] as a validation database. [1] mapped a large number of avalanche outlines using high resolution observations from SPOT6 images. It was found that some avalanche deposits, mainly the large ones, can remain visible several days after an avalanche event [7]. Our detection method is based on "light green color" detections in a multidate SAR image composite in the HSV (Hue Saturation Value) color space. In order to better account for the contribution of snow-free surfaces to SAR signal, we look at the detection results when using different reference images. An automatic classification is applied after a study of the variability of the SAR signal. Our goal is to better understand SAR signal variations during the winter season for different types of land cover and prepare our automatic method of detection. The results of detection are then evaluated against snow avalanche events from the SPOT database of [1]. We also discuss the potential of a Random Forest filtering to reduce false detections. Data and methods used in this study are described in the first Section. In the second Section, we study SAR signal variations in some locations of our test areas. In Section 3, we introduce the color based detection method. The results and evaluations with the SPOT reference are presented in Section 4. Section 5 is the Discussion section and the last section provides conclusions.

\section{Study Context, DAtA And Method}

\section{A. Study Context and Data}

The area under investigation is located in the Swiss Alps, as shown on Fig.1. It is covered by one Sentinel-1 tile, the 32TLS zone. The study area, with, approximately, a surface of 3'800 $\mathrm{km}^{2}$, includes Lower Valais and the southern part of the cantons Vaud and Bern. This area was studied in the work of [7]. Almost 4,000 avalanches were mapped in the 32TLS zone after a period of very high avalanche activity (preceded by several avalanche periods) in January 2018.

We used backscatter coefficients at C-band from SAR observations of Sentinel-1 (noted S1 hereafter). Sentinel-1 mission consists in a constellation of two satellites, Sentinel1A and Sentinel-1B, which were launched in April 2014 and April 2016, respectively, by the European Space Agency (ESA) within the Copernicus Programme. S1 has a sidelooking imaging geometry, so that images are subject to geometry-induced radiometric distortions (shadow, layover and foreshortening effects). We relied on the French Space Agency, CNES (Centre National d'Etudes Spatiales), computing facilities to pre-process $\mathrm{S} 1$ data. The pre-processing included thermal noise removal, speckle filtering using the multi-temporal filtering of [31], radiometric calibration and terrain correction using the SRTM digital elevation model at $30 \mathrm{~m}$. SAR images are obtained for tiles of $110 * 110 \mathrm{~km}^{2} . \mathrm{S} 1$ data are accessible on the PEPS platform. The flowchart of this pre-processing is shown on Fig.A.1.

S1 SAR imagery is not limited by weather conditions ([13], [2]). With two satellites and considering the same incidence
TABLE I

Acquired Sentinel-1 data, with the descending orbit (DES, around 05:43) and ascending orbit (ASC, around 17:22, time UTC).

\begin{tabular}{c|c|c} 
Satellite & Orbit & Date \\
\hline S1A & $139-$ DES & $2018-01-03$ \\
\hline S1B & $088-$ ASC & $2018-01-05$ \\
\hline S1B & $139-$ DES & $2018-01-09$ \\
\hline S1A & $088-$ ASC & $2018-01-11$ \\
\hline S1A & $139-$ DES & $2018-01-15$ \\
\hline S1B & $088-$ ASC & $2018-01-17$ \\
\hline S1B & $139-$ DES & $2018-01-21$ \\
\hline S1A & $088-$ ASC & $2018-01-23$ \\
\hline S1A & $139-$ DES & $2018-01-27$ \\
\hline S1B & $088-$ ASC & $2018-01-29$
\end{tabular}

angle, the revisit frequency is 6 days for each orbit for central Europe. The image acquisition of the descending orbit (D139) is two days ahead of the ascending orbit (A088). There were several high avalanche activity periods in January 2018. To take into account the large areas of SAR geometric distortions not usable for the detection and the differences in dates and times of observation between S1 and SPOT6, we considered the whole month of January for the avalanche detection. Indeed, some deposits from large avalanches released before the period of the 21-23 January were visible several days after the events on the SPOT6 image of the 24 January ([7]). We used all acquisition images of this month with a total of 10 images. Information on these images are introduced on Table I.

In addition, more SAR data was used for selecting the best reference image (here we consider the whole SAR image time serie from early August 2017 to end of December 2017 with a total of 60 SAR images). We mainly focused on SAR backscatter at $\mathrm{VH}$ polarisation. We used the Interferometric Wide Swath (IW) acquisition mode and Level-1 Ground Range Detected (GRD) products.

SPOT mapped avalanches are a great support to evaluate our methods over a rather large area during the exceptional avalanche period of January 2018 in the Alps. As mentioned in Section I, [8] have shown the high potential of SPOT for avalanche mapping using validation points from ground truth $(\mathrm{POD}=0.74)$. The SPOT mapped avalanches cover the majority of the Swiss Alps (surface of 12'500 $\mathrm{km}^{2}$ ) [1]. However, this data set is not exhaustive, some avalanches could not have been mapped because of the visibility and weather conditions but it represents quite well the last avalanche activity of the month of January 2018 (noted SPOT data hereafter).

Using the winter reports and avalanche bulletins made by the WSL Institute for Snow and Avalanche Research SLF, we report some of the meteorological context and danger level of January 2018 for the study zone on Fig.3. In this figure, a timeline of acquisition dates of the SAR and SPOT images is also given. Exceptional quantities of snowfall were measured. In 25 days, at high elevations (above 2'000 m), there was 2,5 to $5 \mathrm{~m}$ of snow. Hence, over a large part of the study 

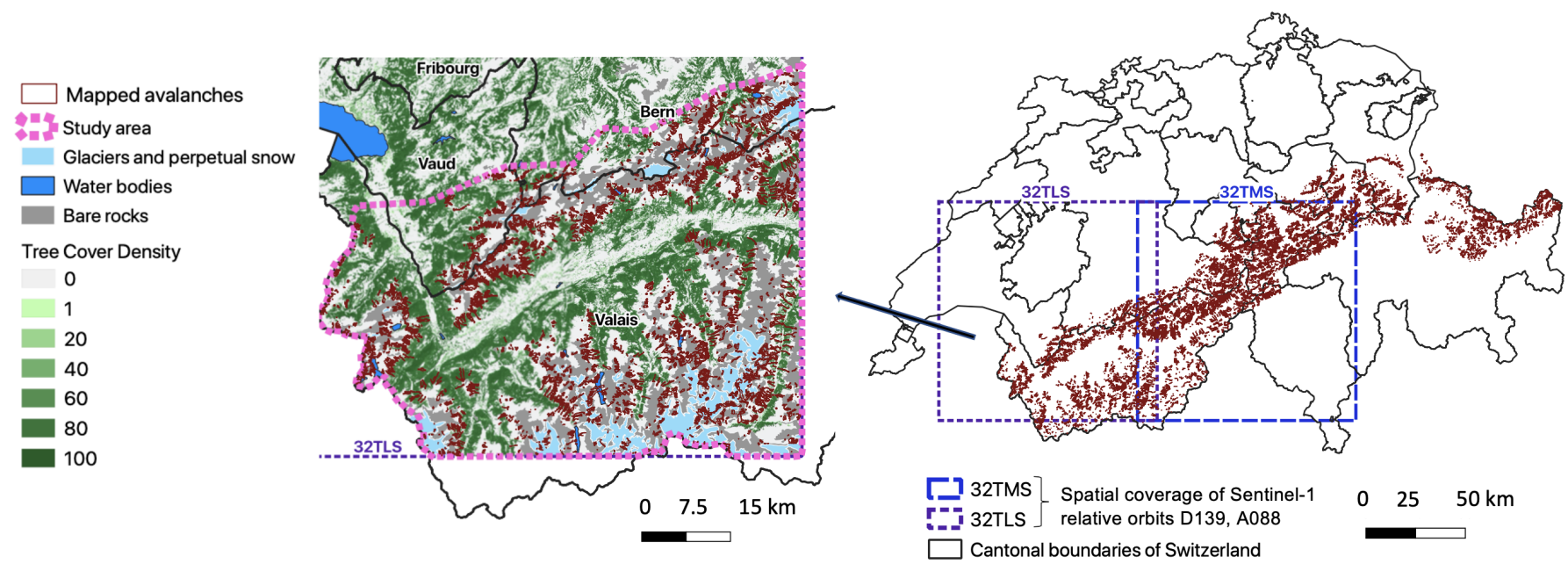

Fig. 1. Location of the study area and the mapped avalanches in the work of [7] and a zoom in of the study area. We use the cantonal boundaries of Switzerland (swissBOUNDARIES3D), the Tree Cover Density layer and the CORINE Land Cover layer (Copernicus Land Cover Services).

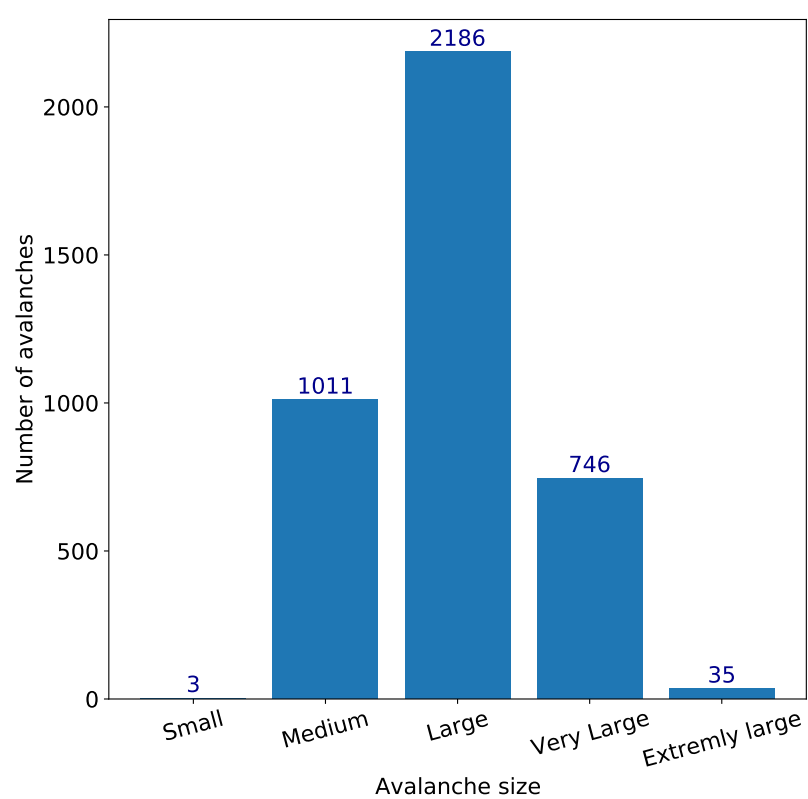

Fig. 2. SPOT mapped avalanches size distributions in the study area.

area, high danger level (level 4) was frequent. The highest danger level occurred on the $22^{\text {nd }}$ of January (level 5). January was also a month incredibly mild with heavy snowfall at high elevation and abundant precipitation at low elevation. Valais was the most impacted zone and many large to extremely large avalanches were reported. The majority of the SPOT mapped avalanches in this area are of large size as shown on Fig.2. Several periods of stormy weather characterised by winds of strong velocity to storm force and heavy to intensive snowfall occurred in January 2018. Numerous snowfall and strong winds predominantly from the West generated large snow accumulations on slopes exposed to the East. Mapped avalanches are mainly located on southeast, east- and south- facing slopes over our study area.

\section{B. Automatic Avalanche debris using color detection}

Fig.4a shows a SAR RGB (Red, Green, Blue) composite image using three SAR acquisitions. In $\mathrm{R}, \mathrm{G}$, they are two successive SAR acquisitions and the image in $\mathrm{B}$ channel is a reference image without snow (R: 20180121, G: 20180127 and B: mean SAR image (average of the images of August and September 2017)). In case of avalanche events, the SAR image after the event will show a localised increase in the backscatter with respect to the acquired image six days earlier. An increase in backscatter appears green, while a decrease in backscatter appears in magenta and areas of no temporal change appear in grey.

The avalanche features, visible in the debris image in Fig.4b, appear as bright green features in the RGB image, which indicates an increase in backscatter with respect to the reference images. These are the kind of characteristics that we wish to detect automatically, without any prior knowledge of the area where the debris might be located. Obviously, a localised increase in the SAR signal can be due to many other reasons besides avalanches. Nevertheless, the signal increase can be considered as a proxy to target areas potentially affected by avalanche debris. The main idea of our approach is therefore to correctly detect areas of bright green, which is equivalent to segmentation in the color space.

To achieve this, we developed a novel method to detect areas of debris using a color space segmentation technique applied on three SAR images acquired at different dates:

- R: the D minus 6 days image (noted D-6 hereafter);

- $\mathrm{G}$ : the activity image (at day $\mathrm{D}$ );

- B: the stable reference image taken several weeks before the event (noted D-M hereafter).

Color based detection methods are widely used in many applications, such as computer graphics, image processing, 

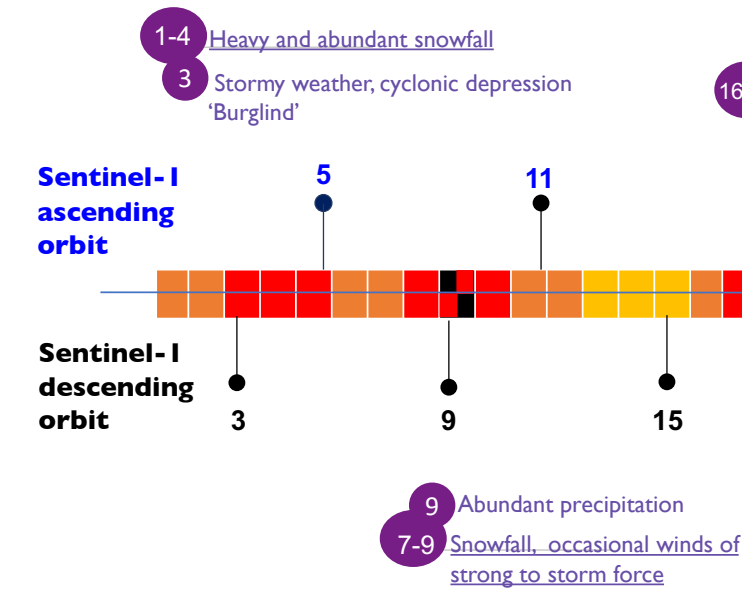

$\longrightarrow$ Timeline of January 2018 , each box corresponds to a day in January and is colored with the highest danger level of the day

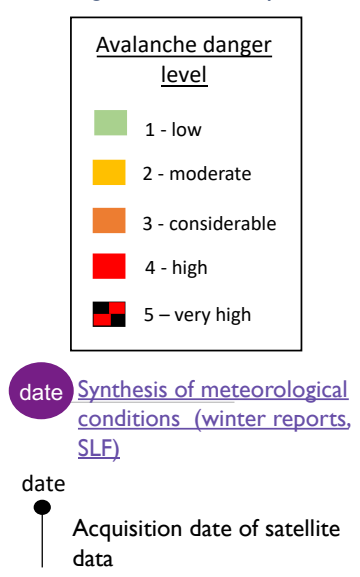

Fig. 3. Synthesis of meteorological conditions, danger level and timeline of the acquisition dates of Sentinel-1 and SPOT6 during January 2018.

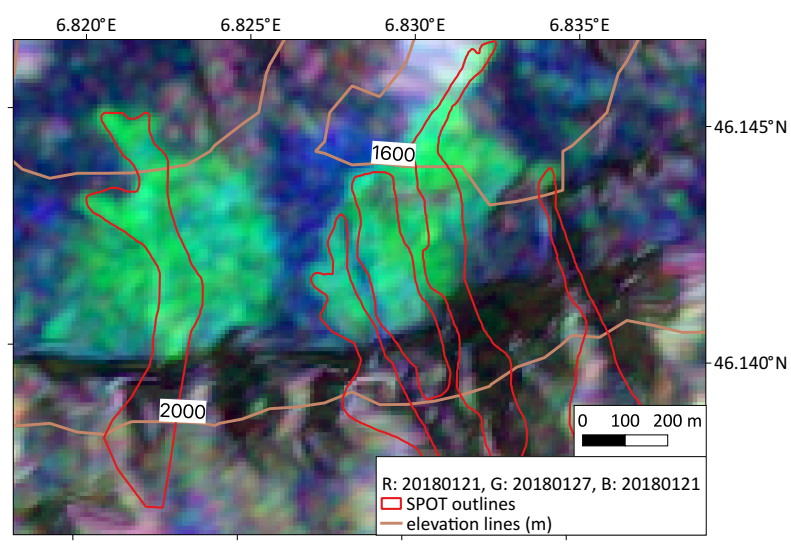

(a)

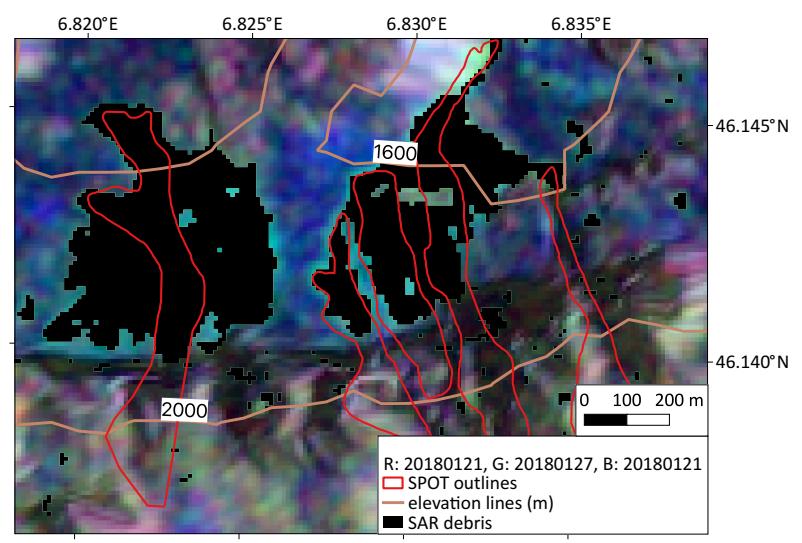

(b)

Fig. 4. Results of the color detection on 4b and RGB composite image on 4a, using R: 20180121, G: 20180127, B: mean summer (average of the images of August and September 2017). We used an adaptive threshold to focus on interesting areas (by using the color space segmentation approach).

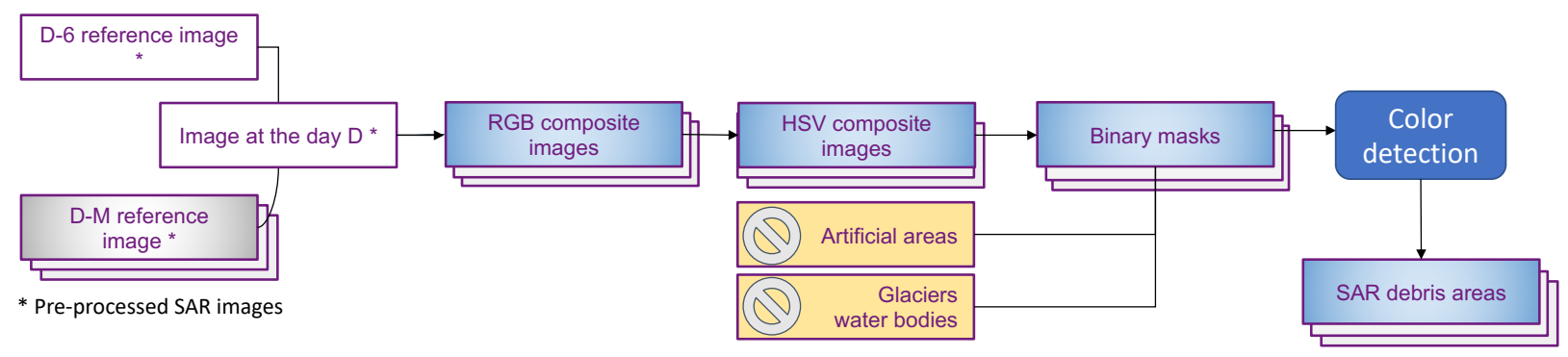

Fig. 5. Flowchart of the automatic method of snow avalanche debris detection.

TV broadcasting, and computer vision. Color space is a mathematical model used to represent color information as three or four different color components. One of the most common color space is RGB (Red Green Blue), where colors are represented in terms of their proportions of red, green, and blue. Another useful color space is HSV (Hue Saturation Value), which is particularly relevant for identifying contrast in images. Our method relies on the use of HSV color space, where the $\mathrm{H}(\mathrm{ue})$ dimension represents the color, the $\mathrm{S}$ (aturation) dimension represents the dominance of this color 
and the $\mathrm{V}$ (alue) dimension represents the brightness. The color detection algorithm seeks for the position of the color and its luminance instead of looking for specific RGB values. The detection of shades of bright green was done in two main steps. (1) First, an RGB composition was made with 3 images (R: D-6 reference image, G: activity image at day D, B: $\mathrm{D}-\mathrm{M}$ reference image). (2) Then, the resulting RGB image was converted into HSV space using the following formulas (provided by Python for the "colorsys" modules):

$$
\begin{gathered}
V=\max (R, G, B) ; \\
S=\left\{\begin{array}{ll}
\frac{\max (R, G, B)-\min (R, G, B)}{\max (R, G, B)}, & \text { if } V \neq 0 \\
0, & \text { otherwise }
\end{array} ;\right. \\
H= \begin{cases}\frac{60(G-B)}{\max (R, G, B)-\min (R, G, B)}, & \text { if } V=R \\
\frac{120+60(B-R)}{\max (R, G, B)-\min (R, G, B)}, & \text { if } V=G \\
\frac{240+60(R-G)}{\max (R, G, B)-\min (R, G, B)}, & \text { if } V=B\end{cases}
\end{gathered}
$$

A green color variation range was chosen to detect several shades of light green. We used OpenCV python open libraries to perform color space conversions. An illustrative example of the detection results is shown in Fig. $4 \mathrm{~b}$ where the detection of light greens are shown in black. One can notice that almost all the light green areas are very well detected by our method. In this example, the D-M reference image is the average of the images taken from August to September 2017. In addition, in layover areas, we observed a scarce number of detections. The flowchart of this method of detection is shown on Fig.5.

It is expected that reference image selection can have a significant impact on the detection results. For instance, the condition of snow on pre-event images can increase false avalanche detection in case of wet to dry snow transition between reference and activity image ([16]). We performed several estimations of debris detections of the same scenes by varying the $\mathrm{D}-\mathrm{M}$ reference images. To select $\mathrm{D}-\mathrm{M}$ reference image dates, we relied on the results of [32] who used different metrics to better select the reference images. Such a reference is commonly used in change detection approaches. In our study, particular emphasis was placed on methods that would allow optimal selection of the D-M reference image. We calculated cross correlations between all SAR image time series, covering the 32TLS tile, from August 2017 to end of January 2018 (ascending/descending images were used separately, which represents a total of 60 SAR images). Cross correlations (1 minus correlation) figures are shown on Fig.A.2 in the Appendix section. To select D-M reference image dates, the idea was to find dates for which a fairly clear loss of correlation can be noted with the observed scenes of January 2018. We aim at better identifying avalanche events. We strive to select D-M reference images that will add information on the surface/ground condition. We also chose D-M reference dates with different meteorological conditions. The correlation matrices were examined to select possible dates for each month between August and November. We ended up with the following reference dates (descending/ascending): $6^{\text {th }} / 8^{\text {th }}$ August 2017, $5^{\text {th }} / 7^{\text {th }}$ September 2017, $5^{\text {th }} / 7^{\text {th }}$ October 2017, $10^{\text {th }} / 12^{\text {th }}$ November 2017. The synthesis of meteorological

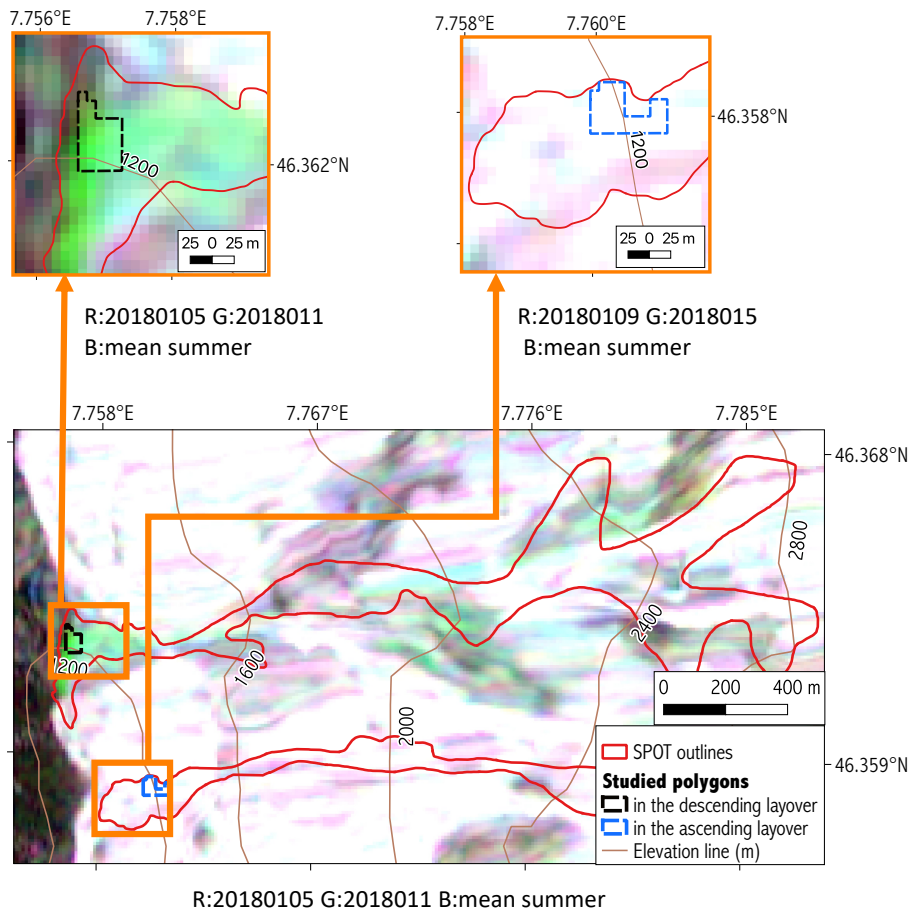

Fig. 6. Localisation of the two polygons of pixels on pastures areas, in different avalanche outlines and different layovers. The background image is an ascending SAR RGB composite.

conditions for each D-M dates is shown on Table II. Moreover, we tested the use of a mean SAR image by averaging the images of August and September 2017. The month of August was particularly warm, with little precipitation. There were many snowfalls in the high mountains during September 2017.

\section{ON SAR SIGNAL VARIABILITY IN A COMPLEX MOUNTAINOUS TERRAIN}

In this section, we examine SAR backscatter variation at the pixel scale in areas associated with different types of land cover : dense forest, glaciers and perpetual snow, bare rocks, and pasture areas. We aim at better understanding the SAR variation at the lowest scale, by studying groups of 35 to 40 pixels.

We selected polygons of pixels located outside and inside avalanche outlines. Polygons located outside SPOT mapped avalanches are on flat areas where no avalanche could occur in order to demonstrate that a high backscatter increase is not connected to other factors outside an avalanche. Groups of pixels validated by the SPOT data are studied so that we can state that the observations acquired at these locations are associated with at least one avalanche event during the month of January 2018. We used the Corine Land Cover (CLC) to get information on the types of land cover and the high resolution layer Tree Cover Density (TCD) to select trees and non-trees covered areas. CLC and TCD of 2018 are produced by the Copernicus Land Cover Services (CGLS) and the European Environment Agency (EEA). The selected pixels are :

- two polygons located in pastures areas, in different avalanche outlines and geometric distortions: one in the 
TABLE II

Selected D-M reference dates and synthesis of meteorological conditions, for both orbits. We used winter reports and avalanche bulletins of the SLF.

\begin{tabular}{|c|c|}
\hline $\begin{array}{c}\text { Date } \\
\text { Descending/Ascending orbit }\end{array}$ & $\begin{array}{l}\text { Meteorological conditions } \\
\text { Descending/Ascending orbit }\end{array}$ \\
\hline $2017-08-06 / 08$ & Little snowfalls in the high mountains \\
\hline $2017-09-05 / 07$ & $\begin{array}{l}\text { Beginning of meteorological autumn } \\
\text { snowfalls in the high mountains }\left(1^{\text {st }}-3^{\text {rd }}\right) / \mathrm{up} \text { to } 10 \mathrm{~cm} \text { of snow above } 3500 \mathrm{~m} \text { (snowfalls the } 6^{\text {th }} \text { and } 7^{\text {th }} \text { ) }\end{array}$ \\
\hline $2017-10-05 / 07$ & Thin snow cover at high altitudes \\
\hline $2017-11-10 / 12$ & $\begin{array}{c}\text { Beginning of winter conditions } \\
\text { Few } \mathrm{cm} \text { of snow/fresh snow and storm winds }\end{array}$ \\
\hline
\end{tabular}

ascending and the other one in the descending path, Fig.6 displays the location of these groups of pixels;

- two other groups of pixels in the same avalanche outline and with different tree cover density: one in a dense forest area (more than $60 \%$ of Tree Cover Density) and another one in a tree-free zone ( $0 \%$ of Tree Cover Density) (location not shown).

- two other groups in bare rock area, one in an avalanche outline and the other one outside SPOT mapped avalanches (location not shown);

- two additional groups in glaciers and perpetual snow area, one in an avalanche outline and the other one outside (location not shown);

The corresponding backscatter time series of the polygons located on different geometric distortion zones (depending on the orbit direction) and SPOT outlines (located on Fig.6), from early December 2017 to end of January 2018, are presented on Fig.7. Results are given for the ascending (Fig.7a) and the descending orbits (Fig.7b). Note that if a pixel falls in the ascending orbit geometric distortion zone, this same pixel will not be affected by the descending orbit distortions, and conversely. As expected, a group of pixels falling into a geometric distortion zone exhibits very high backscatter values: for instance, for the descending orbit, the backscatter average from the polygon falling into the descending layover ranges from around -7.5 to $-2.5 \mathrm{~dB}$ while the signal from the other one (outside the descending layover zone) ranges from -21 to $-17 \mathrm{~dB}$ with an exception on January $15^{\text {th }}$. For the groups of pixels outside layover zones, we can note a lack of significant variation of SAR signal from early December till early January and, for the ascending orbit, strong increases of the signal are observed on January $11^{\text {th }}$ (mean value increases of $10 \mathrm{~dB}$ ) and $29^{\text {th }}$. We do not observe a significant change concerning the signal in layover zones.

We now consider two other groups of pixels on the same SPOT outline, not affected by layovers and associated with dense forest (more than $60 \%$ of Tree Cover Density), tree free ( $0 \%$ of Tree Cover Density), bare rock areas (see Fig.8). For dense forest, the backscatter signal varies smoothly with no significant drop of the signal, except for the decrease on December $12^{\text {th }}$. We observe a rather strong increase in backscatter for tree free pixels on January $11^{\text {th }}$ after avalanche events. We can make the hypothesis that detection over dense forest might be challenging as there was no significant increase for the dense forest polygon because of difficulties to separate the contributions of snow and canopy effects. Similar conclusions can be drawn for bare rock, whose signal variation can be examined with or without avalanche events (see Fig.8b). One could observe a strong increase in backscatter on January $11^{\text {th }}$ while the signal varies more smoothly in areas outside the SPOT outlines.

A decrease of SAR signal can be observed with a dry to wet snow transition. Indeed, snow volume scattering is often more visible in cross-polarization (VH polarization) with a backscatter decrease due to wet snow. It can be linked to absorption, reflection processes, and the decreasing amount of snow volume scattering in a shallowing snowpack ([33]). A net backscatter increase due to wet to dry snow transition can result in false alarms ([16]). The avalanche detection is then impacted and difficult for these periods. Wet snow avalanches are usually easier to detect as they usually have a higher surface roughness than dry snow avalanches ([16], [20]).

We also examine the evolution of the SAR signal on glaciers and perpetual snow areas from early December 2017 to end of January 2018 (not shown). We considered two polygons, one in a SPOT outline and one on flat terrain. For both orbits and polygons, there was no significant SAR variation (not shown). It is worth noting that the mean backscatter range was high in December and January 2018 compared to the reference (the average of August and September 2017), respectively with the ascending and descending orbits: from -14 to $-11 \mathrm{~dB}$ and -12 to $-6 \mathrm{~dB}$ in December and January, while the mean references were around $-18 \mathrm{~dB}$ and $-21 \mathrm{~dB}$. This can be linked to different snow conditions, as discussed previously.

The detection method we have developed is based on the analysis of the evolution of the SAR signal between two successive dates and with the inclusion of a reference image selected with a sensitivity study. We therefore anticipated difficulties in detecting avalanche debris in areas of glaciers, layover and also in areas of dense forest.

In our study, we masked out water bodies, glaciers and perpetual snow, and the artificial areas (urban fabrics, industrial, commercial and transport units, etc.) using classes of land cover. We do not take into account artificial areas as it is rarely affected by snow avalanches. The SAR backscatter variation of glaciers stands out comparing to those of various snow types ([34]). The detection of snow on lakes must be treated differently from snow on open areas ([35]). We choose to exclude water bodies, glaciers and perpetual snow as different studies have found the properties of those surfaces challenging for radar detection and analyzing the signal change would go beyond the scope of this paper ([9], [14], [16]). 


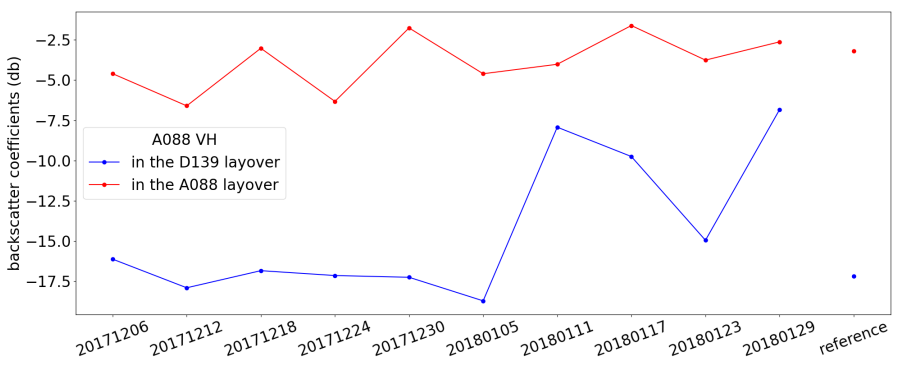

(a) Ascending orbit

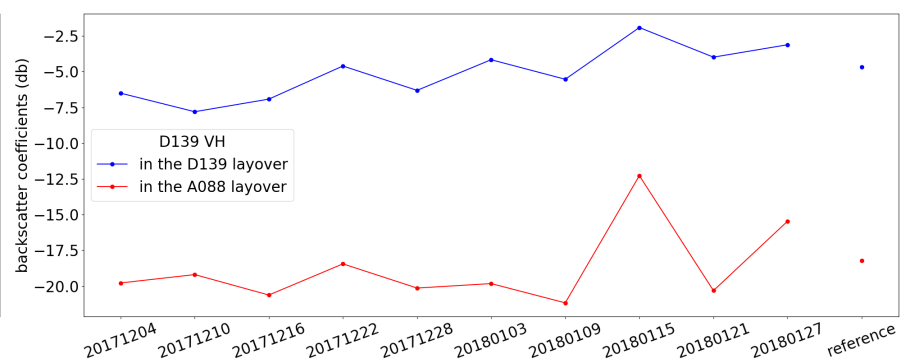

(b) Descending orbit

Fig. 7. Mean backscatter time series from early December 2017 to end of January 2018 for test pixels shown in Fig.6, on a pasture area: with, a, the ascending orbit and b, the descending orbit. The last date named 'reference' is the average of two months (August and September 2017).

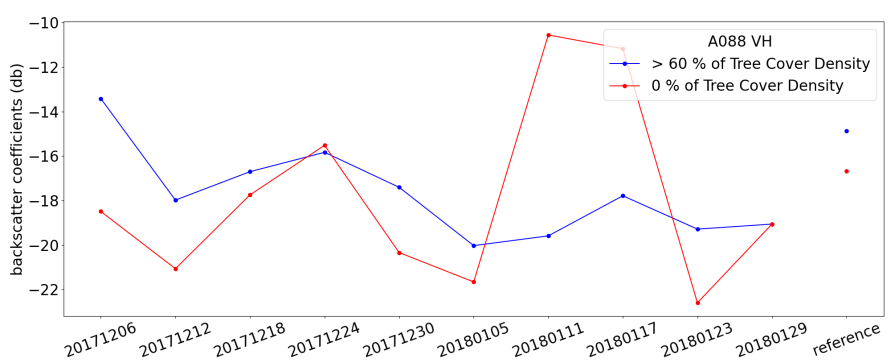

(a) Forest

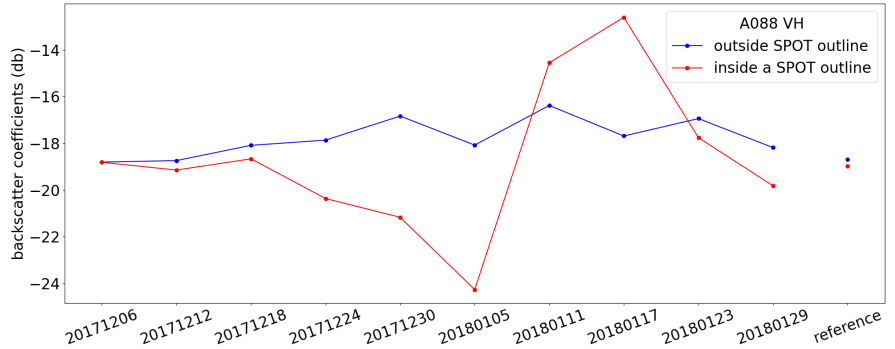

(b) Bare Rock

Fig. 8. Mean backscatter time series from the ascending orbit from early December 2017 to end of January 2018 for polygons on (a) different tree-covered areas, but on the same avalanche outline and (b) on a bare rocks area, inside a SPOT outline and outside, on a flat terrain. The last date named 'reference' is the average of two months (August and September 2017).

\section{Avalanche Debris Detection Results}

We used our detection method to calculate binary products of avalanche debris for the entire month of January 2018 (using 5 SAR images from the ascending orbit and 5 images from the descending orbit). To facilitate comparison with the SPOT reference, a binary product was generated by assigning a value of 1 to any pixel that was detected as debris, 0 if no detection was performed. All Sentinel-1 acquisitions during January were accounted for. We obtained as many binary products as D-M reference images that were tested. We recall that the following dates were used as D-M reference images (ascending/descending): $6^{\text {th }} / 8^{\text {th }}$ August 2017, $5^{\text {th }} / 7^{\text {th }}$ September $2017,5^{\text {th }} / 7^{\text {th }}$ October $2017,10^{\text {th }} / 12^{\text {th }}$ November 2017 and a mean image using all images of August and September 2017.

We then studied the properties of the avalanche debris pixels using the SPOT data, which we consider here as our reference. We first calculated the number of pixels falling within a SPOT contour and also the location of SPOT outlines for which no debris was detected. We also examined the characteristics of SAR detection outside the SPOT outlines.

\section{A. Overall statistics}

In this section, we consider the detection results obtained using SAR images of the average of August and September as D-M reference for both orbits.

We introduce some global statistics with rates of True Positive (noted TP hereafter) and False Negative detection (noted FN hereafter). A snow avalanche event is considered TP if at least 10 SAR debris pixels fall in the SPOT outline. In case of lack of SAR detection or when less than 10 pixels are inside the SPOT mapped avalanche, the SPOT outline is then associated with a FN event. The True Positive Rate (TPR) is the percentage of TP events compared to TP and FN events. The False Negative Rate (FNR) is the percentage of FN compared to the TP and FN events. Thus, TPR and FNR of 100 and $0 \%$, respectively, are for perfect detections. The choice of 10 pixels is made considering the low spatial resolution of $\mathrm{S} 1$ and the fact that many studies highlighted the difficulty to detect snow avalanches of small sizes using S1 (size 1, 10 to $500 \mathrm{~m}^{2}$, according to the European Avalanche Warning Services, EAWS) ([16], [20]). [16] used a cut-off minimum size of 10 pixels to reduce false alarms and pointed out the limitation of detecting small avalanches using S1 images. [20] also noticed that small avalanches were often missed when using S1.

With the SPOT outlines, the TPR are around $58 \%$ and 36 $\%$ with the ascending and descending orbits, respectively. By combining the detection from both orbits, we obtain a TPR of around $66.4 \%$.

Fig.9 shows percentages of FNR and TPR using both orbits per classes of orientations. The maximum values of FNR are obtained with South-Western and Western orientations for the ascending orbit (FNR are around 69 to $63 \%$ ) and Eastern, South-Eastern and North-Eastern orientations for the descending orbit (around 83 to $70 \%$ ). This is in line with the presence of areas of geometric distortion affecting the 


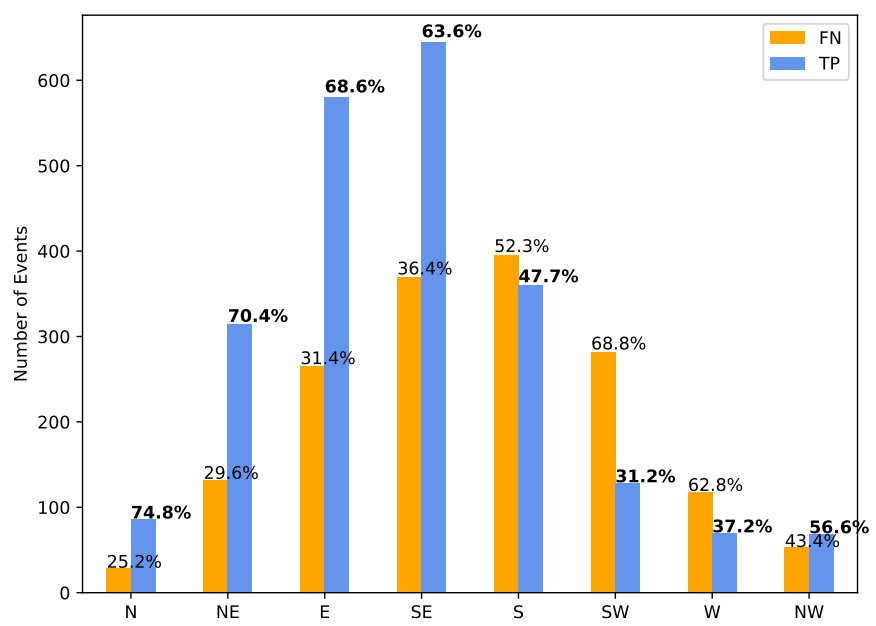

(a) Ascending orbit

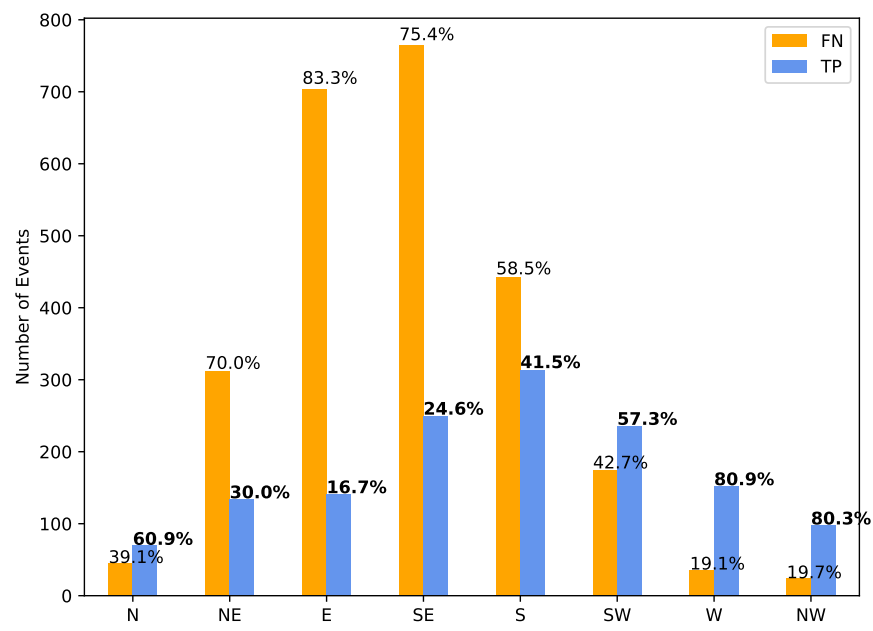

(b) Descending orbit

Fig. 9. The percentages of TPR and FNR using the ascending and the descending orbits, with the average of two months for the D-M reference image. We are looking at these percentages per classes of orientations. For each orientation, TPR and FNR of 100 and $0 \%$, respectively, are for perfect detections.

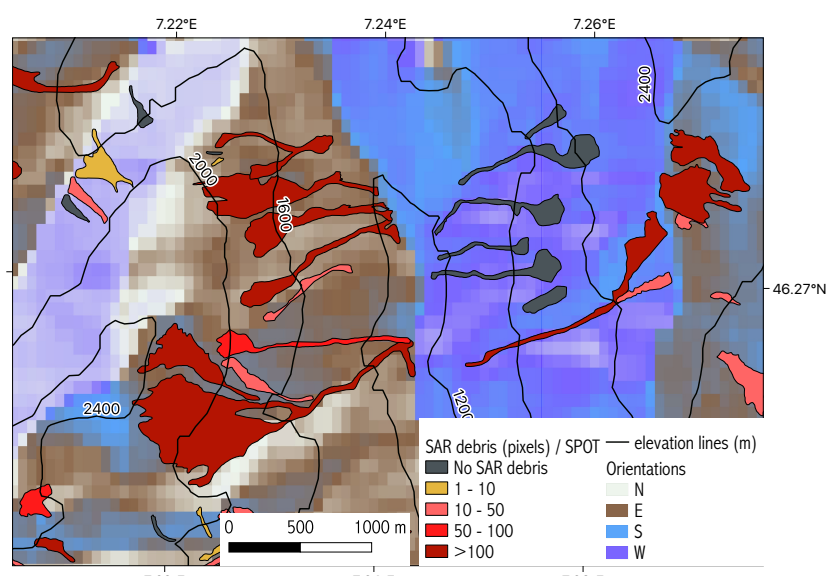

(a) Ascending orbit

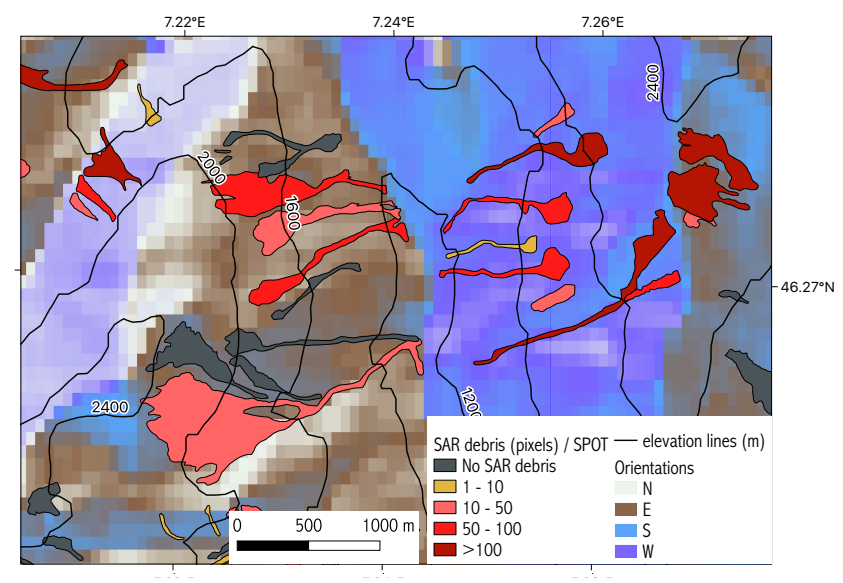

(b) Descending orbit

Fig. 10. Number of debris pixels in SPOT mapped avalanches as shades of red: the darker the color, the larger the number of debris pixels within an avalanche detected outline. Grey indicates avalanche tracks where no debris is detected by SAR. Yellow indicates a small number of SAR debris, while dark red represents a high number of SAR pixels. Results are given for : a, the ascending orbit and b, the descending orbit. We used the average of two months for the D-M reference image. The background map is the classes of orientation (SRTM digital terrain model).

Eastern orientation for the descending orbit and the Western orientation for the ascending orbit. Indeed, we can observe higher TPR in Western orientation for the descending orbit than for the ascending one. We can see it visually on Fig.10. This figure shows the SPOT mapped avalanches as shades of red depending on the number of detected pixels falling in each outline: the darker the color, the larger the number of debris pixels within an avalanche outline. Note that outlines with less than 10 SAR pixels detected (yellow and grey colors) are not considered in our statistics.

We now consider percentages of TPR and FNR per classes of avalanche size. We used the same classification as in the study of [7] and we excluded the smallest class (size 1). The remaining avalanche events are classified as : "Medium: size 2" (501 to $\left.10,000 \mathrm{~m}^{2}\right)$, "Large: size 3" $\left(10,001\right.$ to $\left.80,000 \mathrm{~m}^{2}\right)$, "Very large: size 4" (80,001 to 500,000 m²) and "Extremely large: size 5" (greater than 500,000 $\mathrm{m}^{2}$ ) events. We expect to have less detection of size 2 avalanches as we have chosen not to consider SPOT outlines with less than 10 detected pixels. Approximately $30 \%$ of our detections of less than 10 pixels and with at least 1 pixel, fall in the outlines associated with avalanche size $2(29.8 \%$ and $40 \%$ obtained in ascending orbit with a reference image taken in November and early August respectively). Unsurprisingly, the larger the avalanche, the easier SAR detection is (at least 10 pixels) and the larger is the number of detected pixels that fall within the avalanche boundary. Thus, about 21 to $13 \%$ of medium-sized avalanches are detected with ascending and descending orbits respectively. The percentages are 64 to $36 \%$ for large avalanches, 87 to 63 $\%$ for very large avalanches and 100 to $94 \%$ for extremely large events. 
TABLE III

Percentages of TPR using different datasets of SAR debris detection (using different D-M reference dates and only one D-6 reference image) for both orbits. TPR are calculated with respect to the SPOT mapped avalanches. A SPOT avalanche detected event is a SPOT outline that includes at least 10 detected pixels.

\begin{tabular}{c|c|c|c|c}
\multirow{2}{*}{ D-M reference image } & \multicolumn{2}{|c}{ Whole January } & \multicolumn{2}{c}{ End of January } \\
& DES & ASC & DES & ASC \\
\hline Aug-Sep 2017 & 35.7 & 57.8 & 23.6 & 33.5 \\
\hline 6-8 Aug 2017 & 44.6 & 59.1 & 32.6 & 34.8 \\
\hline 5-7 Sep 2017 & 42.2 & 68.0 & 30.3 & 47.8 \\
\hline 5-7 Oct 2017 & 44.6 & 64.4 & 34 & 42.4 \\
\hline 10-12 Nov 2017 & 47.1 & 71.7 & 37.3 & 52.9 \\
\hline D-6 image & 30.1 & 59.8 & 12.4 & 29.3
\end{tabular}

\section{B. Results using different $D$-M reference images}

We now consider the whole set of debris calculations using various selections of D-M reference images. Table III shows the percentages of TPR and FNR for, respectively, the ascending and the descending orbits using different datasets of SAR debris detection. Results using the whole month of January and the end of the month are compared on this table. We use the following D-M reference dates (descending/ascending): $6^{\text {th }} / 8^{\text {th }}$ August $2017,5^{\text {th }} / 7^{\text {th }}$ September $2017,5^{\text {th }} / 7^{\text {th }}$ October 2017, $10^{\text {th }} / 12^{\text {th }}$ November 2017 . FNR and TPR are calculated with respect to the SPOT mapped avalanches. On Table III, we note that for the D-M reference images, the use of selected individual dates improves detection results with regards to the average over two months. Considering the whole month of January: with the descending and ascending orbits, respectively, around 36 to $58 \%$ of SPOT avalanche events are detected with the average of August and September while the maximum of detection is obtained using the 10 and $12^{\text {th }}$ of November, with 47 to $72 \%$ of detected SPOT avalanche events. If we use the same method of detection but with only two images, the D-6 reference image (in $\mathrm{R}$ and $\mathrm{G}$ channels) and the activity image, we obtain similar results than when using the average over two months as the D-M reference image : 30 to $60 \%$ of SPOT avalanche events are detected, respectively, with the descending and ascending orbits for all of January 2018. Therefore, the use of two reference images (D-6 and a reference image) appears to improve debris detection results. The average reference image $\mathrm{D}-\mathrm{M}$ mixes dates that might not be as favorable for detection (warm weather and few snowfalls/rain in the high mountains). Moreover, choosing D-M reference dates with similar snow and meteorological conditions to the beginning of winter season seems to enhance SAR detections (November dates). We also note that there is more debris detection with the evening orbit (ascending) than with the morning orbit (descending). For the events verified with SPOT mapped avalanches, we also have a high percentage of TPR with the evening orbit while having a fairly low percentage of FNR compared to the morning orbit.

Comparing the results obtained for the whole month and those obtained for the end of January, we observe, respectively for the ascending and descending orbits, average gaps of around $23 \%$ and $12 \%$ (see Table III).

We studied the results using different D-M reference images

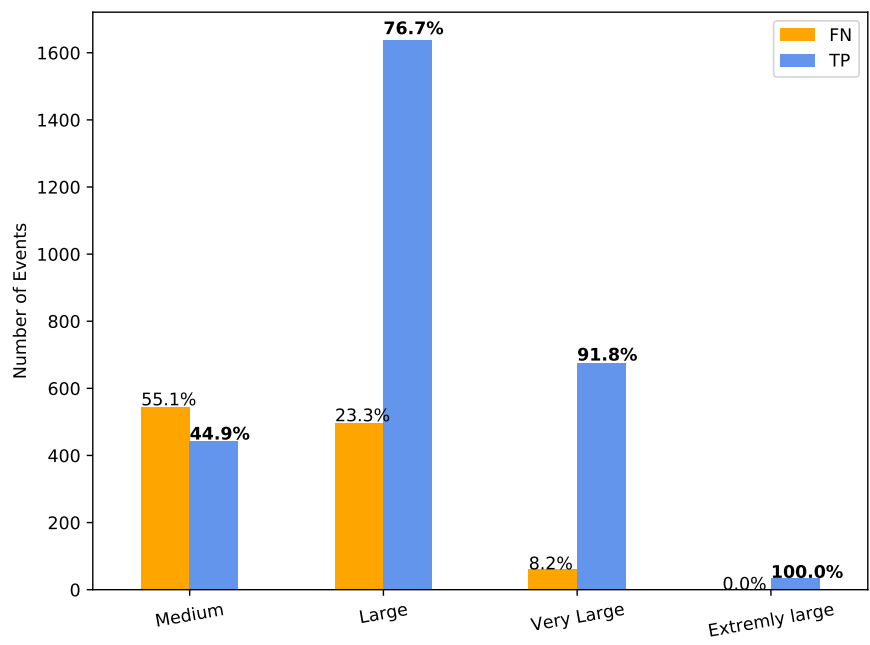

Fig. 11. The percentages of TPR and FNR per classes of avalanche size using the ascending orbit, with the $12^{\text {th }}$ November 2017 for the D-M reference image. For each avalanche size, perfect detections are obtain with a TPR of $100 \%$ and a FNR of $0 \%$.

per classes of orientations for the whole month of January. The general trend in detection results by orientation is broadly similar regardless of the D-M reference chosen. For the ascending orbit, we note better detection rates for the North, North East, East, South, South East orientations while the South West, West and North West orientations are worse. This effect can be explained by the impact of geometric distortions. Considering a given orientation, the best detections are obtained with the reference of $12^{\text {th }}$ November 2017 with the highest TPR and the lowest FNR. For the South East orientation, which is associated with the highest number of SPOT avalanche events, the TPR/FNR are, respectively, 78.7 / $21.3 \%$ when the D-M reference is that of November 12 compared to rates of $65.9 / 34.1 \%, 74.8 / 25.2 \%, 70.4$ / 29.6 and 63.6/36.4\% when the D-M reference is that of August 8, September 7, October 7 and Summer mean image respectively. For the descending orbit, the South orientation is associated with the highest number of SPOT avalanche events. The TPR are respectively : $52.1,52.1,52.6,54$ and $41.5 \%$ when the D-M reference is that of August 6, September 5, October 5, November 10 and Summer mean image respectively.

We also looked at avalanche detection results by avalanche size and for different reference images for the month of January. Medium/large/very large avalanches seem to be better detected when using individual dates rather than a mean summer image (best results are obtained with September and November images). For example, for the ascending orbit, the detection rate of "Large" and "Very large" avalanches increases, respectively, from $64 \%$ and $87 \%$, when D-M is the summer average, to $77 \%$ and $92 \%$ when the $12^{\text {th }}$ November reference is used (see Fig.11). Poorer detection results are obtained for the medium avalanche class. Therefore, the selection of reference images based on the criterion of cross correlation of images seems to be relevant to improve avalanche debris detection. November was the beginning of winter but with different ground conditions from January. 


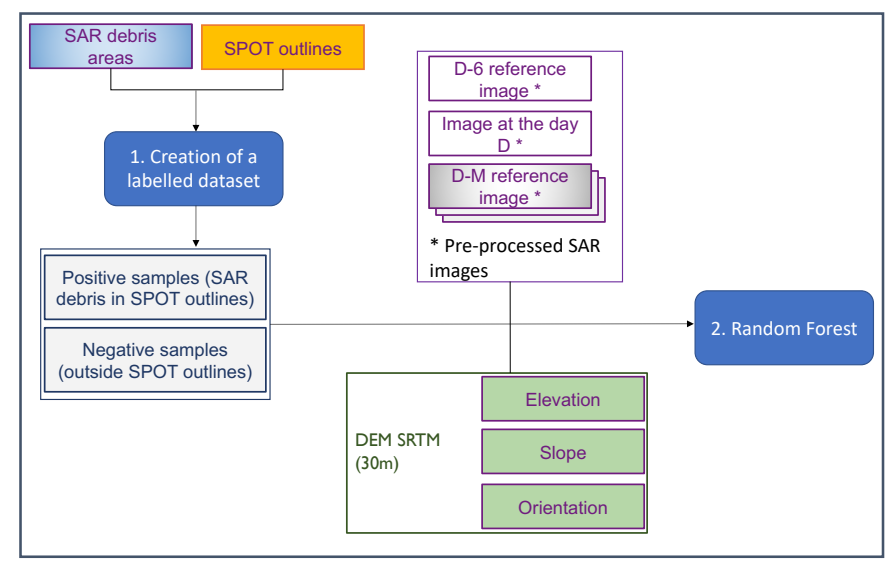

(a) Random Forest filtering

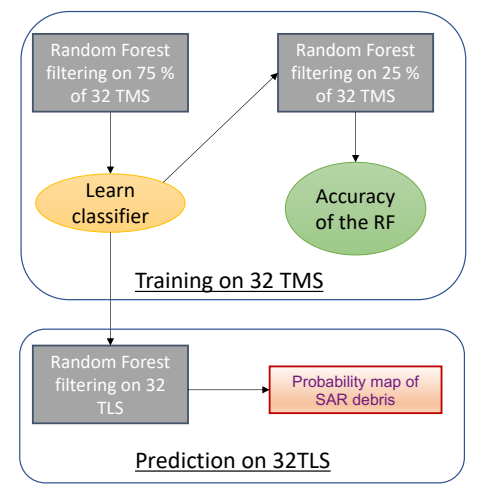

(b) Training and Prediction

Fig. 12. General steps of Random Forest filtering used for the training and prediction of SAR avalanche debris.

\section{Potential of a Random Forest approach : Improving the detection by filtering with machine learning}

The complexity of the interaction between the radar signals and the snow is also linked to the terrain characteristics such as surface type, slope, orientation and elevation. Our debris detection method targets areas with a strong increase in backscatter between two successive dates. However, an increase in signal may be due to other effects than the debris of a snow avalanche. This is why we additionally developed and compared an automatic filtering of the color-based detection using a machine learning approach. The goal is to accurately remove the false debris detections by learning the non-linear function linking the SAR signal variation between successive dates, a D-M reference image and the topology information (slope, orientation, elevation and tree cover) to the presence or absence of avalanche deposit.

We trained a random forest (noted RF hereafter) classification model on the 32TMS tile (see Fig. 1) in order to avoid any overfitting on the 32TLS tile, where the results are then evaluated. RF is an ensemble learning method that constructs multiple decision trees during training and averages the regression prediction over the trees ([28]). Each tree is independent and generates a class prediction. The best class prediction is selected at the end (vote for the most popular class) ([28]). Moreover, a machine learning algorithm can be robust to label noise (i.e. mislabelled samples). Thanks to the SPOT mapped avalanches on the 32TMS, we created a labelled database from which we trained our RF model: for each of the January SAR acquisition dates, the color detection-based SAR detections that were in agreement with the SPOT mapped detections were labelled as positive samples. Conversely, the SAR detections outside of the SPOT outlines were labelled as negative samples. For the training, we took the same number of positive and negative samples in order to facilitate the learning. Machine learning can learn even if the training data is not perfect. As discussed in Section II-A3, SPOT6-7 images are a reliable tool to map avalanches. However, a minority of avalanches could not have been mapped because of the visibility and weather conditions. There are also differences in dates and times of observation between Sentinel-1 and SPOT. Thus, some samples could have been mislabelled. From a grid search analysis on $75 \%$ of the 32TMS tile detections, we selected a RF model with 128 trees of depth 10 . Grid search allowed us to select the best parameters for the RF classification : we tested different values for each parameter and selected the best combination of settings. We obtained on the remaining $25 \%$ of the 32TMS tile a $81 \%$ accuracy for the descending D066 orbit and $95 \%$ accuracy for the ascending A088 orbit. Once trained (few minutes), the model is computationally efficient and the prediction output of the full 32 TLS is estimated in less than a minute. Among the outputs of the RF classification is the probability of occurrence of events, which varies from 0 to 1 , which is associated with detections. False detections can be reduced by only considering detections associated with a high probability (e.g. $>0.7$ ). We can thus derive a probability map for each SAR acquisition date for January 2018, which gives a confidence for every detection. Two flowcharts summarize the described processing steps: the Random forest filtering is shown on Fig.12a and the steps of the training and the prediction are introduced on Fig.12b.

Table IV introduces the percentages of TPR using different thresholds for each orbit and the average of two months for the D-M reference image. Concerning the RF filtering, we did not add a minimum value of number of detected pixels as we suppose that the RF filters SAR debris according to the non-linear function linking the S1 data and the topology information. By default, we consider that a pixel belongs to an avalanche if the probability is above 0.7 for the descending orbit and above 0.2 for the ascending orbit. We empirically chose these values because the RF results are below the SAR " $\geq 10$ pixels in each SPOT track" results. In addition, outside and inside SPOT outlines, the higher the threshold, the more pixels are filtered. This is why we can observe a drop of TPR values with a high threshold. Moreover, visually, there is an improvement of the detection, as it removed many isolated pixels and kept the majority of verified detections.

An example is shown on Fig.13 with the detected pixels using : a, the color detection-based method, $b$, the probability of RF output being an avalanche and c the RF filtering on 


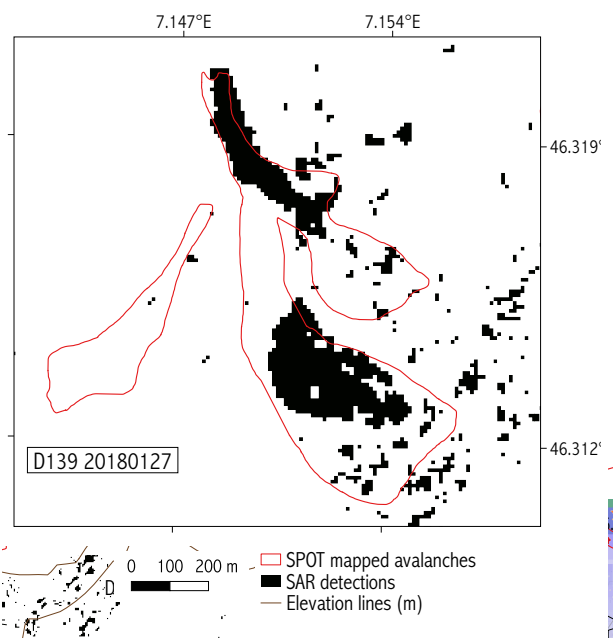

(a)

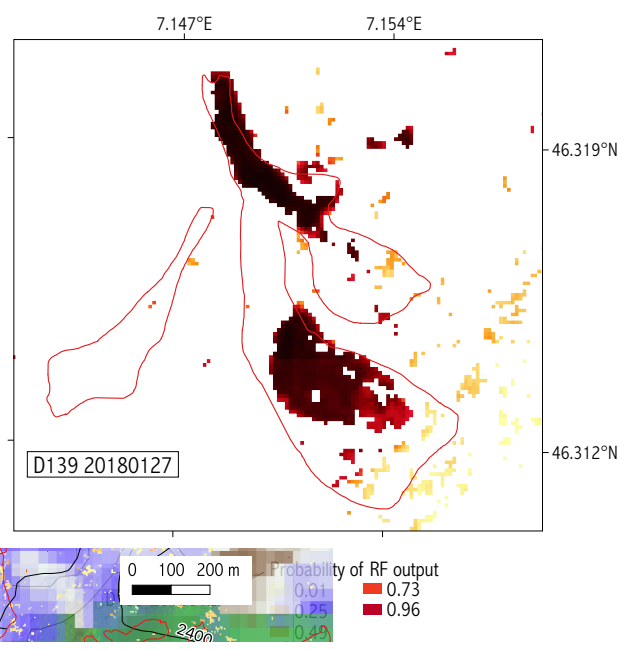

(b)

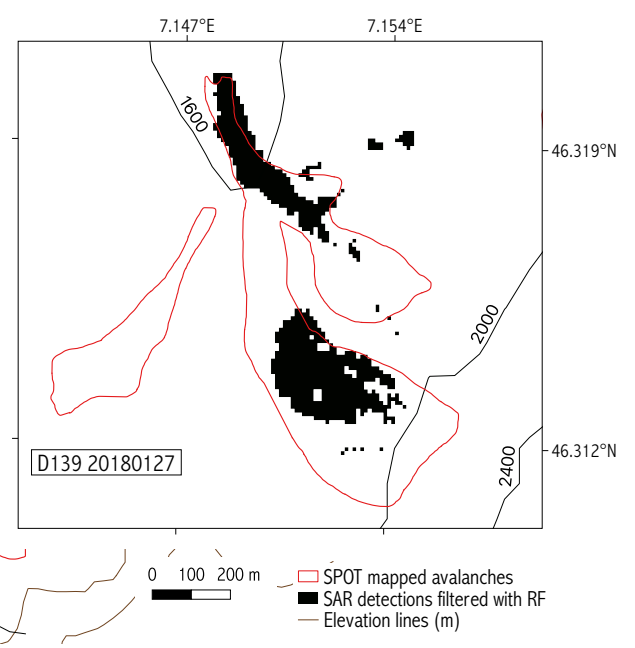

(c)

Fig. 13. Example of detected pixels with : a, SAR and c, RF methods using the D139 acquisition date, the 27 January 2018. The probability of RF output of being an avalanche is shown in $b$.

TABLE IV

Percentages of TPR using different thresholds of RF filtering. We filtered all SAR debris.

\begin{tabular}{c|c|c|}
$\begin{array}{c}\text { SAR filtered debris } \\
\text { Threshold of RF }\end{array}$ & $\begin{array}{c}\text { Ascending } \\
\text { orbit }\end{array}$ & $\begin{array}{c}\text { Descending } \\
\text { orbit }\end{array}$ \\
\hline RF $>0.1$ & 68.1 & 53.4 \\
\hline RF $>0.2$ & 38.1 & 52.2 \\
\hline RF $>0.3$ & 25.3 & 50.5 \\
\hline RF $>0.7$ & 8.2 & 31.3
\end{tabular}

January $27^{\text {th }}$ (descending orbit). Note that the RF filtering shown in Fig.13c was obtained using 0.7 as threshold.

We compared results obtained with different RF thresholds (not shown). We can note that the RF does not deteriorate results of validated pixels using SAR color based detection. For January 2018, the number of detected pixels outside the SPOT outlines with no RF filtering are around 1'048'160 / 1'479'500 with the descending and ascending orbits, respectively. With a RF filter, the number of pixels decreases and we obtained : with a RF threshold above 0.3 , around 587'500 / 99'500 and with a RF filter above 0.7 , around 157 ' $100 / 8$ ' 500 .

We can observe with the descending and ascending orbits, respectively, that the number of pixel outside the SPOT outlines is significantly decreased compared to the number of pixel inside the SPOT paths: with a RF filter of 0.3, 56 / $7 \%$ of detected pixels are kept outside SPOT outlines while $88 / 25 \%$ of detected pixels inside SPOT outlines are kept by the RF filtering, respectively. The difference between orbit RF filtering models have not been investigated, but might be due to the number and variety of available training data in the 32TMS. The RF filtering reduced the number of pixels outside SPOT mapped avalanches, while retaining a majority of the true positives ones, without removing verified detections. We trained the RF classification model with the SPOT mapped avalanches, thus we expected a reduction of the number of pixels outside SPOT mapped avalanches.
On the one hand, for detected pixels falling inside the SPOT-mapped avalanches, the orientation distribution after RF filtering is close to the distribution of verified detections (not shown). On the other hand, for detected pixels falling outside the SPOT-mapped avalanches, RF filtering makes some changes to the detections according to the orbit direction. For the descending orbit, RF filtering significantly reduces the number of detected pixels for west orientations compared to east orientations. For instance, with an RF filter of 0.3, $7 \%$ (69\%) of the detected pixels on the eastern (western) slopes are discarded after filtering.

\section{DISCUSSION}

Regarding the detection method, we use a color space segmentation approach to detect areas of avalanche debris. For this purpose, we rely on the use of two reference images (D-6 and D-M) instead of one, which was used in previous studies (D-6 or D-12) ([2], [36], [37] [14], [16], [8], [20]). It is worth noting here that some studies dealing with wet snow detection using Sentinel-1 have investigated the issue of using not only one but two reference images. A method has been proposed by [38] which suggests using two reference images taken before and after the melting season. However, if the second reference image is without snow and the ground was already drying, false detection can occur ([39]).

We looked at the impact of using the D-M images by performing debris detection with only the D-6 image as reference. The results are summarized in Table III where one could see that the use of two reference images improves the percentages of TPR by about 6 to $17 \%$ for the ascending orbit and by about 3 to $12 \%$ for the descending orbit (values depend on D-M images). These results also suggest that the D-M image must be carefully selected to maximise debris detection. We have shown that the use of an average image as a D-M reference does not seem to be the right option for optimising avalanche debris detection, but rather the selection of reference images. The D-M reference images were selected 
using the criterion of low cross-correlation of SAR images with respect to the avalanche activities periods of January 2018. By studying the meteorological and snow conditions of these dates, and comparing it to the obtained results, it seems that best results are obtained when using a reference image at the very beginning of winter. This was the case of the ascending D-M reference date of the $12^{\text {th }}$ November 2017 (considerable snowfalls and beginning of the winter condition), for which we obtained the highest detection score. During the $10^{\text {th }}$ November 2017, the snow depth was lower than the $12^{\text {th }}$ November (11-13 November was a snowfall period). This change of snow conditions could explain the difference of results using the ascending and descending orbits. Good results were obtained using September reference images. The issue of reference images still requires further work to select the best possible option. The use of images at well selected dates would allow better capture of ground/snow conditions and effect of growing vegetation. The D-6 and D images in January 2018 were also different for both orbits : images from the descending orbit are acquired in the early morning while those of the ascending orbit are acquired, two days after, in the end of the afternoon. The snow and meteorological conditions are then also different and lead to different results. The extent of the distortion zones (which do not allow the detection of debris on specific mountain slopes) could also partly explain the detection gaps between the two orbits. Further studies are underway to better understand these differences in detection.

In the study of [8], it was emphasized that S1 detection was often partial. Indeed, the detected areas can be disconnected as many patches corresponding to a single avalanche. Our detection is done at the pixel level and not at the level of polygons or avalanche shapes. We evaluated our results by looking at detected events verified by SPOT outlines (with at least 10 pixels falling within a mapped avalanche). Contrary to studies that generated avalanche polygons, we can't evaluate avalanche detection outside SPOT tracks in absence of other validation datasets ([16], [8]). A specific processing to combine the different patches into a single avalanche should allow better detection performance. It would avoid overestimating the number of avalanches or underestimating their size.

We have relied on the previous work of [8] and [7] who showed the very good quality of the SPOT database which we have considered as our reference. In [1], the authors have documented the avalanche period of January 2018 over a large spatial extent using SPOT6's high resolution measurements by mapping 18,737 avalanche contours in Switzerland providing us with a great tool to evaluate our algorithms. The SPOT database is very relevant for us since it helps us to evaluate several properties of avalanches, including avalanche size, we could hardly find elsewhere with such high spatial coverage. The current study covers one month with successive avalanche periods and must necessarily be extended with further assessments covering different periods and areas, with various meteorological conditions and zones of different characteristics. The use of SPOT images should also be complemented as far as possible by in-situ avalanche observations.

SPOT mapped avalanches were identified after the avalanche period from the 21 to 23 January 2018. In our study, we focus on January 2018 which had several successive avalanche periods. Results of the detections with the Sentinel-1 images taken at the end of January show average differences in detections of the order of 12-23\% compared to the detections of all S1 images of the month (see Table III). Regarding the use of the SPOT data set, [7] estimated that 10 to $20 \%$ of the avalanches were released before the avalanche period of the end of January, with an upper limit value of $25 \%$ of old avalanches. Including the whole month of January could be useful to include as much as possible avalanches from previous avalanche periods that might still have been visible and therefore mapped in the SPOT reference. This choice may lead to some bias if we consider avalanches that occurred in early January and are not present in the SPOT reference. Using multiple images could also increase the cumulative number of false detections and will not improve avalanche detection in areas of geometric distortion.

We expected to find different results for the SAR and SPOT avalanche monitoring because of the relatively large areas not taken into account in the SAR images due to geometric distortions and the differences in dates and times of observation between Sentinel-1 and SPOT. It is worth noting that studies of [20] and [8] already compared the Sentinel-1 manual detection to SPOT6-7 mapping and found different percentages of detection. The gap between these detection scores can be explained by the difference of visibility, spatial resolution and acquisitions dates (variation of the snow pack state and avalanche visibility according to the meteorological conditions changes) between the optical and radar satellites. [20] found that S1 detected $68 \%$ of SPOT detected avalanches while SPOT detected $44 \%$ of S1 mapped avalanches. [8] detected with S1 $55 \%$ of SPOT avalanches and, with SPOT mapping, $89 \%$ of S1 avalanches were detected. Mapped avalanches were linked to validation points from ground truth. With the $10^{\text {th }}$ and $12^{\text {th }}$ November 2017 as D-M reference images, we also found different detection scores: 48 and $72 \%$ of SPOT mapped avalanches, with the descending and ascending orbits of S1 respectively.

In the Section IV, we discussed the detection of SPOT avalanche events using our segmentation method. It is worth noting that we applied a detection threshold of 10 pixels in a outline so that it could be considered in our statistics. Relaxing the 10 pixels threshold criterion can be of interest in situations where avalanche pixels can be detected at the edges of the SPOT outlines (due to differences in acquisition dates between SPOT and Sentinel-1, changes in meteorological conditions, etc.). Another effect of using the 10 pixels threshold is the potential deterioration of the detection of small and medium-sized avalanches (sizes 1 and 2). We have examined the predominant avalanche size associated with outlines for which there are less than 10 SAR pixels detected (and at least 1 pixel). Whatever the D-M reference chosen, avalanche 
size 2 was found to be the dominant size (more than 30 $\%$ of cases). This may partly explain the poorer detection results for medium avalanches. This result is consistent with those of [8], who noted a similar tendency to under detect small and medium sized avalanches. [8] showed that there was a clear improvement in detection scores with increasing size of avalanches (12\% of medium avalanches, $42 \%$ of large avalanches, $90 \%$ for very large ones and $100 \%$ for extremely large events). Despite differences in detection rates, we obtained relatively similar trends with our detection method with better detection with increasing avalanche size $(21 \%$ (45 $\%$ ) of medium-sized events, $64 \%(77 \%)$ of large ones, $87 \%$ $(92 \%)$ of very large ones, $100 \%(100 \%)$ of extremely large events with the mean summer for the D-M reference image (the $12^{\text {th }}$ November 2017 for the D-M reference image) and the ascending orbit).

As noted in [8], the avalanche debris mapped in the Sentinel-1 data often appear as multiple patches. In our paper, the event definition method allows us to consider even fragmented debris. We consider that we get an avalanche event if at least 10 pixels of SAR debris fall within a SPOT contour. This may also explain the relatively higher scores we get.

It is worth noting that the classes of avalanche size of our detected events are based on the area of SPOT outlines and not the volume or destructive potential of avalanches.

Another useful information of SPOT mapped avalanches is the quality of outlines. Extending the SPOT mapped avalanches could be helpful in taking better account of the pixels detected on the edges and it may also be useful for situations where the detection of avalanches on the SPOT image is difficult, leading to a lower quality indicator (presence of clouds, shadows, ....).

For the mountain regions of interest, the choice of auxiliary data such as DEM (and its resolution) is not obvious. In our study, our choice was driven by the use of the CNES generic processing chain which can be used worldwide and the availability of the $30 \mathrm{~m}$ SRTM DEM model. In the framework of surface type classification, [40] have investigated the influence of the DEM employed for terrain normalization of backscatter and coherence data. The authors show that high-resolution TanDEM-X DEM (20 or 30 $m$ resolution) was the global DEM providing the largest reduction of terrain induced variability. It would be very useful to conduct such an analysis with the specific objective of detecting avalanche debris.

Snow conditions can have a significant impact on Sentinel1 debris detection results. It is often expected that wet snow avalanches are easier to detect overall (e.g. [20], [16]). Indeed, as it is reminded in the work of [41], with C-band SAR images, there are physical based limitations to the detection of dry snow avalanches. Nevertheless, [8] show good detection results in dry snow especially for medium to large avalanches. In case of highly changing conditions like in January 2018, it is possible that the radar backscatter images before and after the event show fast snow transitions/changes making debris detection considerably more difficult. For instance, from 15 to 23 January, the snowfall line oscillated around 500 to 2'000 m (winter reports of the SLF). There were intense snowfalls and periods of lower avalanche danger with no precipitations. With stable dry snow conditions, avalanches can be more easily detected (see for instance, [8] and [16]). [16] obtained better results for dry to wet snow transition than with stable wet snow conditions or with wet to dry snow transition. All this argues for examining the results of detection under diverse snow conditions.

Another direction of improvement of our system is to take into account the local incidence angle of the SAR as it was the case in the [20], [8] and [22] studies. In our study, we rely on the Sentinel-1 processing chain of the CNES to process SAR time series. This processing chain does not yet include a module to correct or attenuate layover/foreshortening effects but the method of [42] which allows terrain flattening is scheduled to be implemented in future updates of the system.

Since our assumption for detecting avalanche pixels is based only on areas with a significant increase in backscatter compared to reference images, we expect to overestimate the number of pixels labelled as avalanche debris. Outside the SPOT corridors we are testing the potential of a RF filtering approach to remove false detections. The idea is to train the filtering model on detections that we can evaluate (with the SPOT database) and then apply it to areas for which we have no evaluation data. The learning process takes into account labelled SAR detections as well as terrain characteristics. We therefore expect this method to help reduce false detections, but work remains to be done to properly calibrate the method (define best probability threshold values, increase training data, take into account different avalanche periods, etc.).

\section{CONCLUSIONS}

In this study, we developed a novel method to automatically detect areas of snow avalanche debris using a color space segmentation technique applied to SAR image time series. We provide the first study on SAR detection using D-6 and D$M$ reference dates. We have shown that, in our study area, almost $66 \%$ of the avalanche events recorded in the SPOT database were detected by our method, by combining the ascending and descending orbits and using the average of two months as the D-M reference image. Small avalanches were not detected by SAR images and, depending on the orientation of the terrain, some avalanches are not detected by either the ascending or the descending orbit. Large, very large and extremely large avalanches were mainly detected, in agreement with [8] who also noted a clear improvement in detection scores with increasing size of avalanches: $64 \%$ of avalanches of size 3 are detected, $87 \%$ of size $4,100 \%$ of size 5 , with the mean summer for the D-M reference image and the ascending orbit. We have also shown that detection results vary with the selected reference image; best detection results are obtained with individual dates chosen in autumn with $72 \%$ of verified avalanche events using the ascending orbit. Poorer results were obtained with the descending orbit; and we do 
not yet have a precise explanation for this. This detection gap could be linked to the difference between the state of the snow pack/ground conditions of D-M reference dates of the ascending and descending orbits. At first, we selected D$\mathrm{M}$ reference images with the lowest cross-correlation values with respect to the avalanche activities periods of January 2018. Reference images taken during autumn seem to give best results of detection. The comparison of results obtained using only one D-6 images and using two references image (D-M and D-6) demonstrates the potential of selecting specific D-M reference dates (increase of TPR by about 6 to $17 \%$ for the ascending orbit and by about 3 to $12 \%$ for the descending orbit).

A possible improvement of the detection method was briefly presented in order to discuss its potential and possible future developments. We have tested an automatic filtering system to remove false detections using a Random Forest classification model. Despite the preliminary status of this machine learning filtering, its results are very promising.

Our approach generates avalanche detection at a pixel level. Thus, we cannot directly compare our results with those of studies that detect avalanche polygons. The evaluation of the number of avalanches detected by our method outside SPOT data is difficult, as we do not produce avalanche polygons and the detection can be partial [8]. Therefore, more improvements of our method need to be done.

More in-depth studies are underway to generate systematic validation of the color based detection method and optimize the detection scores. An interesting direction could be the exploitation of a series of reference images rather than a single one with statistical metrics to derive probabilities of avalanche debris occurrences. Methods will be further evaluated on a larger spatial scale including the French mountains and for several avalanche periods with different meteorological conditions.

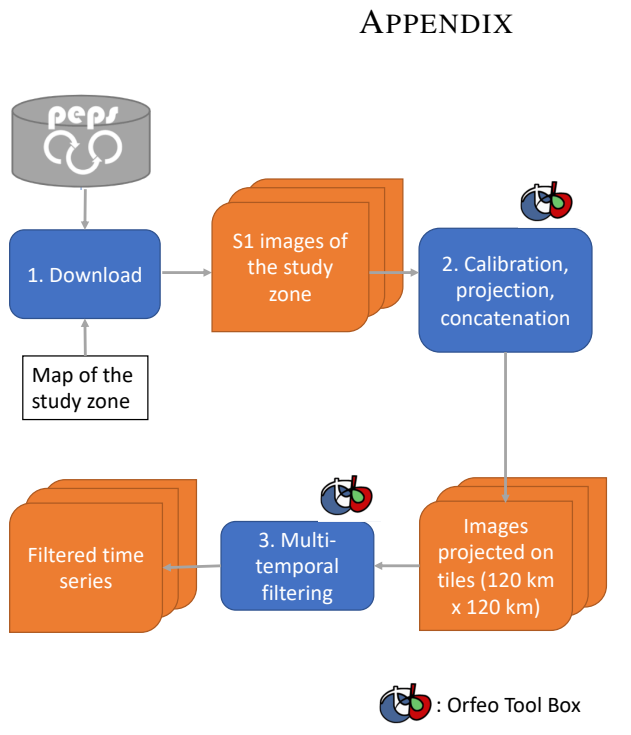

Fig. A.1. Flowchart of the pre-processing of Sentinel-1 images using S1Tiling chain developed by the CNES radar service.

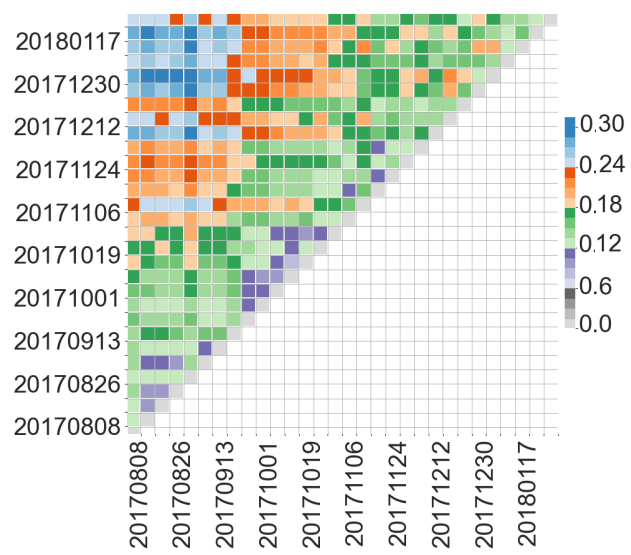

(a) Ascending orbit

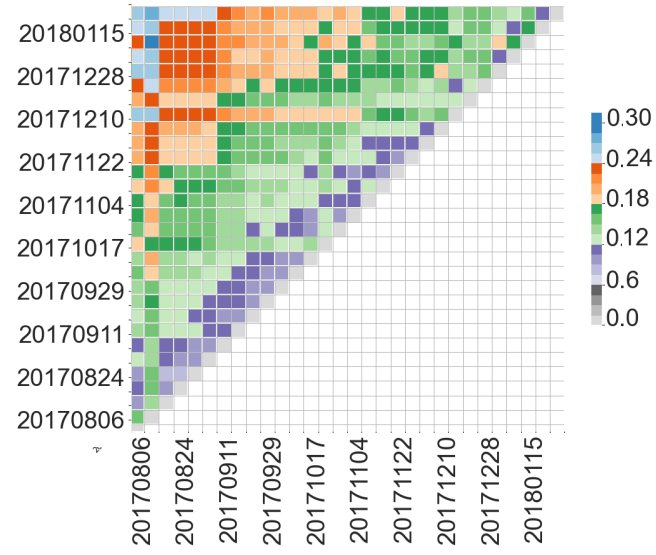

(b) Descending orbit

Fig. A.2. Cross correlations of backscatter coefficients between all SAR time series, covering the 32TLS tile, for both orbits, from August 2017 to the end of January 2018. The values are equal to: 1- Corr, with Corr the values of backscatter correlations. 


\section{ACKNOWLEDGMENT}

The authors would like to deeply thank two anonymous reviewers for their valuable comments and suggestions. Mapped avalanche outlines from SPOT images were realised by [1]. We wish to thank all co-authors of [1] for sharing the data. The authors would also like to acknowledge:

- The support provided by the PNTS French program (Programme National de Télédétection Spatiale).

- The support provided by CNES for preprocessing SAR images using the PEPS facilities.

- The support provided by the Research and Service Support team of the European Space Agency (ESA) for the processing resources and data access provided for the pre-processing of Sentinel-1 images through the RSS CloudToolbox service. The support of the Geohazard Exploitation Platefom (GEP) initiative through the project approved by ESA for monitoring avalanche deposits over the French mountains.

Meteorological data are available and provided by the WSL Institute for Snow and Avalanche Research (SLF): https://www.slf.ch/en/avalanche-bulletin-and-snowsituation/weekly-and-winter-reports.html. Cantonal boundaries of Switzerland (swissBOUNDARIES3D) are produced by the Federal Office of Topography, swisstopo and available at : https://opendata.swiss/fr/dataset/swissboundaries3dkantonsgrenzen. The Tree Cover Density and CORINE Land Cover were produced by the Copernicus Global Land Service (CGLS) and the European Environment Agency (EEA) "with funding by the European Union".

\section{REFERENCES}

[1] E. Hafner and Y. Bühler, "Spot6 avalanche outlines 24 january 2018," EnviDat, 2019.

[2] E. Malnes, M. Eckerstorfer, and H. Vickers, "First sentinel-1 detections of avalanche debris," The Cryosphere Discuss, no. 9, pp. 1943-1963, 2015.

[3] Y. Bühler, A. Huni, M. Christen, R. Meister, and T. Kellenberger, "Automated detection and mapping of avalanche deposits using airborne optical remote sensing data," Cold Regions Science and Technology 57, pp. 99-106, 2009.

[4] F. R. Lato, M.J. and Y. Buhler, "Automated detection of snow avalanche deposits: segmentation and classification of optical remote sensing imagery," Natural Hazards Earth System Sciences, p. 2893-2906, 2012.

[5] S. R. Larsen S $\varnothing$, Salberg A, "Automatic avalanche mapping using texture classification of optical satellite imagery," edited by: Lasaponara, R., Masini, N., and Biscione, M., EARSeL, 2013,Towards Horizon 2020, p. 399-410, 2013.

[6] K. Kozreniowska, Y. Buhler, M. Marty, and O. Korup, "Regional snowavalanche detection using object-based image analysis of near-infrared aerial imagery," Natural Hazards and Earth System Sciences, vol. 17, pp. 1823-1836, 2017.

[7] Y. Bühler, E. D. Hafner, B. Zweifel, M. Zesiger, and H. Heisig, "Where are the avalanches? rapid spot6 satellite data acquisition to map an extreme avalanche period over the swiss alps," The Cryosphere, vol. 13, p. 3225-3238, 2019.

[8] E. D. Hafner, F. Techel, S. Leinss, and Y. Bühler, "Mapping avalanches with satellites - evaluation of performance and completeness," The Cryosphere, vol. 15, p. 983-1004, 2021.

[9] M. Eckerstorfer and E. Malnes, "Manual detection of snow avalanche debris using high-resolution radarsat-2 sar images," Cold Regions Science and Technology, vol. 120, no. 6, pp. 205-218, 2015.

[10] A. Wiesmann, U. Wegmuller, M. Honikel, T. Strozzi, and C. L. Werner, "Potential and methodology of satellite based sar for hazard mapping," Proceedings of IGARSS, Sydney, Australia, pp. 9-13, 2001.
[11] F. Ulaby, R. Moore, and A. Fung, "Microwave dielectric properties of natural earth materials," Microwave remote sensing, vol. 3, pp. 20172027, 1986.

[12] E. Malnes, M. Eckerstorfer, and A. Jonsson, "Remote sensing of avalanches in northern norway using synthetic aperture radar," International Snow Science Workshop Grenoble, 2013.

[13] C. Bühler, Y. ans Bieler, C. Pielmeier, A. Wiesmann, R. Caduff, R. Frauenfelder, C. Jaedicke, and G. Bippus, "All-weather avalanche activity monitoring from space ?" International Snow Science Workshop ISSW, At Banff, AB, Canada, 2014.

[14] D. S. Wesselink, E. Malnes, M. Eckerstorfer, and R. c. Lindenbergh, "Automatic detection of snow avalanche debris in central svalbard using c-band sar data,," Cold Regions Science and Technology, vol. 120, no. 6, pp. 205-218, 2017.

[15] M. Eckerstorfer, E. Malnes, H. Vickers, and K. Muller, "Operational avalanche activity monitoring using radar satellites: From norway to worldwide assistance in avalanche forecasting," International Snow Science Workshop, At Innsbruck, Austria, 2018.

[16] M. Eckerstorfer, H. Vickers, E. Malnes, and J. Grahn, "Near-real time automatic snow avalanche activity monitoring system using sentinel-1 sar dara in norway," Remote Sensing, vol. 11, pp. 773-791, 2019.

[17] M. Eckerstorfer, E. Malnes, and U. Domaas, "Avalanche debris detection using satellite-borne radar and optical remote sensing," International Snow Science Workshop 2014, 2014.

[18] H. Vickers, M. Eckerstorfer, E. Malnes, and A. P. Doulgeris, "Synthetic aperture radar (sar) monitoring of avalanche activity: An automated detection scheme," 20th Scandinavian Conference, SCIA, Troms $\varnothing$, Norway. Proceedings, Part II. Springer International Publishing, 2017.

[19] M. Eckerstorfer, K. Müller, J. Grahn, E. Malnes, R. Engeset, T. Humstad, A. Widforss, and D. Moldestad, "Norway's operational avalanche activity monitoring system using sentinel-1," Virtual Snow Science Workshop (poster), 2020.

[20] S. Leinss, R. Wicki, S. Holenstein, S. Baffelli, and Y. Bühler, "Snow avalanche detection and mapping in multitemporal and multiorbital radar images from terrasar-x and sentinel-1," Natural Hazards and Earth System Sciences, vol. 20, pp. 1783-1803, 2020.

[21] D. Small, "Sar backscatter multitemporal compositing via local resolution weighting," IGARSS - 2012 IEEE International Geoscience and Remote Sensing Symposium, 25 Munich, Germany, 22.07.2012 27.07.2012, pp. 4521-4524, 2012.

[22] C. Tompkin and S. Leinss, "Backscatter characteristics of snow avalanches for mapping with local resolution weighting," IEEE Journal of Selected Topics in Applied Earth Observations and Remote Sensing, 2021.

[23] F. Karbou, C. Coleou, M. Lefort, M. Deschartres, N. Eckert, R. Martin, C. G., and A. Dufour, "Monitorin avalanche debris in the french mountains using observations form sentinel-1 satellites," Proceedings, International Snow Science Workshop, Innsbruck, Austria, 2018.

[24] P. E. Kummervold, E. Malnes, M. Eckerstorfer, I. M. Arntzen, and F. Bianchi, "Avalanche detection in sentinel-1 radar images using convolutional neural networks," International Snow Science Workshop, At Innsbruck, Austria, 2018.

[25] A. U. Waldeland, J. H. Reksten, and A.-B. Saldberg, "Avalanche detection in sar images using deep learning," IGARSS 2018 - IEEE International Geoscience and Remote Sensing Symposium, 2018.

[26] S. Sinha, S. Giffard-Roisin, a. M. D. F. Karbou, N. Eckert, A. Karas, C. Coleou, and C. Monteleoni, "Can avalanche deposits be effectively detected by deep learning on sentinel-1 satellite sar images," International Workshop on Climate Informatics, 2019.

[27] F. M. Bianchi, J. Grahn, M. Eckerstorfer, E. Malnes, and H. Vickers, "Snow avalanche segmentation in sar images with fully convolutional neural networks," IEEE Journal of Selected Topics in Applied Earth Observations and Remote Sensing, 2020.

[28] L. Breiman, "Random forests," Machine Learning 45 (1), p. 5-32, 2018.

[29] J. B. Hamar, A.-B. Salberg, and F. Ardelean, "Automatic detection and mapping of avalanches in sar images," IEEE International Geoscience and Remote Sensing Symposium (IGARSS), vol. 130, p. 689-692, 2016.

[30] P. D. Sielenou, L. Viallon-Galinier, P. Hagenmuller, P. Naveau, S. Morin, M. Dumont, D. Verfaillie, and N. Eckert, "Combining random forests and class-balancing to discriminate between three classes of avalanche activity in the french alps," Cold Regions Science and Technology, vol. 187,2021

[31] S. Q. J.J. Yu, "Multi-channel filtering of sar images," IEEE Transactions on Geoscience and Remote Sensing, 2001.

[32] F. Karbou, G. James, P. Durand, and A. Atto, Thresholds and distances to better detect wetsnow over mountains with Sentinel-1 SAR image time 
series. ISTE-WILEY Science- Change Detection and Image TimeSeries Analysis, 2020, in prep, ch. 5.

[33] H. Lievens, M. Demuzere, H. Marshall, R. H. Reichle, L. Brucker, P. de Rosnay, M. Dumont, M. Girotto, W. Immerzeel, T.Jonas, and al, "Snow depth variability in the northern hemisphere mountains observed from space," Nat. Commun., vol. 4629, 2019.

[34] H. Rott, "Possibilities and limits of synthetic aperture radar capabilities for snow and glacier monitoring," Advances in Space Research, vol. 4, pp. 241-246, 1984.

[35] E. Malnes and T. Guneriussen, "Mapping of snow covered area with radarsat in norway," IEEE Xplore, 2002.

[36] D. S. Wesselink, "Optimisation of methods for snow avalanche detection in sar images," Delf University of Technology, Science in Geoscience and Remote Sensing, 2015.

[37] M. Eckerstorfer, E. Malnes, and K. Muller, "A complete snow avalanche activity record from a norwegian forecasting region using sentinel-1 satellite-radar data," Cold Regions Science and Technology, 2017.

[38] J. Koskinen, J. J. Pulliainen, and M. Hallikainen, "The use of ers-1 sar data in snow melt monitoring," Remote Sensing, vol. 35, pp. 601-610, 1997.

[39] K. Luojus, J. Pulliainen, S. Metsamaki, and M. Hallikainen, "Snowcovered area estimation using satellite radar wide-swath images," IEEE Trans. Geosci. Remote Sens., vol. 45, pp. 978-989, 2007.

[40] I. Borlaf-Mena, M. Santoro, O. Badea, and M.A., "Investigating the impact of digital elevation models on sentinel-1 backscatter and coherence observations," Remote Sensing, vol. 12/18, p. 3016, 2020.

[41] M. Eckerstorfer, K. Mueller, E. Malnes, and H. D. Oterhals, "Manual and automoatic detection of dry snow avalanches in sentinel-1 sar images," IEEE Conference, IGARSS, 2021.

[42] D. Small, "Flattening gamma: radiometric terrain correction for sar imagery," IEEE Transactions on Geoscience and Remote Sensing, vol. 49, pp. 8, 3081-3093, 2011. 